\title{
TESTS OF CONSERVATION LAWS
}

\section{Updated June 2006 by L. Wolfenstein and T.G. Trippe.}

In keeping with the current interest in tests of conservation laws, we collect together a Table of experimental limits on all weak and electromagnetic decays, mass differences, and moments, and on a few reactions, whose observation would violate conservation laws. The Table is given only in the full Review of Particle Physics, not in the Particle Physics Booklet. For the benefit of Booklet readers, we include the best limits from the Table in the following text. Limits in this text are for $\mathrm{CL}=90 \%$ unless otherwise specified. The Table is in two parts: "Discrete Space-Time Symmetries," i.e., C, P, $T, C P$, and $C P T$; and "Number Conservation Laws," i.e., lepton, baryon, hadronic flavor, and charge conservation. The references for these data can be found in the the Particle Listings in the Review. A discussion of these tests follows.

\section{$C P T$ INVARIANCE}

General principles of relativistic field theory require invariance under the combined transformation $C P T$. The simplest tests of $C P T$ invariance are the equality of the masses and lifetimes of a particle and its antiparticle. The best test comes from the limit on the mass difference between $K^{0}$ and $\bar{K}^{0}$. Any such difference contributes to the $C P$-violating parameter $\epsilon$. Assuming $C P T$ invariance, $\phi_{\epsilon}$, the phase of $\epsilon$ should be very close to $44^{\circ}$. (See the review " $C P$ Violation in $K_{L}$ decay" in this edition.) In contrast, if the entire source of $C P$ violation in $K^{0}$ decays were a $K^{0}-\bar{K}^{0}$ mass difference, $\phi_{\epsilon}$ would be $44^{\circ}+90^{\circ}$.

Assuming that there is no other source of $C P T$ violation than this mass difference, it is possible to deduce that[1]

$$
m_{\bar{K}^{0}}-m_{K^{0}} \approx \frac{2\left(m_{K_{L}^{0}}-m_{K_{S}^{0}}\right)|\eta|\left(\frac{2}{3} \phi_{+-}+\frac{1}{3} \phi_{00}-\phi_{\mathrm{SW}}\right)}{\sin \phi_{\mathrm{SW}}},
$$

where $\phi_{\mathrm{SW}}=(43.51 \pm 0.05)^{\circ}$, the superweak angle. Using our best values of the $C P$-violation parameters, we get $\left|\left(m_{\bar{K}^{0}}-m_{K^{0}}\right) / m_{K^{0}}\right| \leq 10^{-18}$ HTTP://PDG.LBL.GOV 
at $\mathrm{CL}=95 \%$. Limits can also be placed on specific $C P T$-violating decay amplitudes. Given the small value of $\left(1-\left|\eta_{00} / \eta_{+-}\right|\right)$, the value of $\phi_{00}-\phi_{+-}$ provides a measure of $C P T$ violation in $K_{L}^{0} \rightarrow 2 \pi$ decay. Results from CERN[1] and Fermilab[2] indicate no $C P T$-violating effect.

\section{$C P$ AND $T$ INVARIANCE}

Given $C P T$ invariance, $C P$ violation and $T$ violation are equivalent. The original evidence for $\mathrm{CP}$ violation came from the measurement of $\left|\eta_{+-}\right|=\left|A\left(K_{L}^{0} \rightarrow \pi^{+} \pi^{-}\right) / A\left(K_{S}^{0} \rightarrow \pi^{+} \pi^{-}\right)\right|=(2.236 \pm 0.007) \times 10^{-3}$. This could be explained in terms of $K^{0}-\bar{K}^{0}$ mixing, which also leads to the asymmetry $\left[\Gamma\left(K_{L}^{0} \rightarrow \pi^{-} e^{+} \nu\right)-\Gamma\left(K_{L}^{0} \rightarrow \pi^{+} e^{-} \bar{\nu}\right)\right] /[\mathrm{sum}]=(0.332 \pm 0.006) \%$. Evidence for $C P$ violation in the kaon decay amplitude comes from the measurement of $\left(1-\left|\eta_{00} / \eta_{+-}\right|\right) / 3=\operatorname{Re}\left(\epsilon^{\prime} / \epsilon\right)=(1.66 \pm 0.26) \times 10^{-3}$. In the Standard Model much larger $C P$-violating effects are expected. The first of these, which is associated with $B-\bar{B}$ mixing, is the parameter $\sin (2 \beta)$ now measured quite accurately to be $0.725 \pm 0.037$. A number of other $C P$-violating observables are being measured in $B$ decays; direct evidence for $C P$ violation in the $B$ decay amplitude comes from the asymmetry $\left[\Gamma\left(\bar{B}^{0} \rightarrow K^{-} \pi^{+}\right)-\Gamma\left(B^{0} \rightarrow K^{+} \pi^{-}\right)\right] /[\mathrm{sum}]=-0.113 \pm 0.020$. Direct tests of $T$ violation are much more difficult; a measurement by CPLEAR of the difference between the oscillation probabilities of $K^{0}$ to $\overline{K^{0}}$ and $\overline{K^{0}}$ to $K^{0}$ is related to $T$ violation [3]. Other searches for $C P$ or $T$ violation involve effects that are expected to be unobservable in the Standard Model. The most sensitive are probably the searches for an electric dipole moment of the neutron, measured to be $<6 \times 10^{-26} \mathrm{e} \mathrm{cm}$, and the electron $(0.07 \pm 0.07) \times 10^{-26} e \mathrm{~cm}$. A nonzero value requires both $P$ and $T$ violation.

\section{CONSERVATION OF LEPTON NUMBERS}

Present experimental evidence and the standard electroweak theory are consistent with the absolute conservation of three separate lepton numbers: electron number $L_{e}$, muon number $L_{\mu}$, and tau number $L_{\tau}$, except for 
the effect of neutrino mixing associated with neutrino masses. Searches for violations are of the following types:

a) $\Delta L=\mathbf{2}$ for one type of charged lepton. The best limit comes from the search for neutrinoless double beta decay $(Z, A) \rightarrow(Z+2, A)+e^{-}+e^{-}$. The best laboratory limit is $t_{1 / 2}>1.9 \times 10^{25} \mathrm{yr}(\mathrm{CL}=90 \%)$ for ${ }^{76} \mathrm{Ge}$.

b) Conversion of one charged-lepton type to another. For purely leptonic processes, the best limits are on $\mu \rightarrow e \gamma$ and $\mu \rightarrow 3 e$, measured as $\Gamma(\mu \rightarrow e \gamma) / \Gamma(\mu \rightarrow$ all $)<1.2 \times 10^{-11}$ and $\Gamma(\mu \rightarrow 3 e) / \Gamma(\mu \rightarrow$ all $)<$ $1.0 \times 10^{-12}$. For semileptonic processes, the best limit comes from the coherent conversion process in a muonic atom, $\mu^{-}+(Z, A) \rightarrow e^{-}+(Z, A)$, measured as $\Gamma\left(\mu^{-} \mathrm{Ti} \rightarrow e^{-\mathrm{Ti}}\right) / \Gamma\left(\mu^{-} \mathrm{Ti} \rightarrow\right.$ all $)<4 \times 10^{-12}$. Of special interest is the case in which the hadronic flavor also changes, as in $K_{L} \rightarrow e \mu$ and $K^{+} \rightarrow \pi^{+} e^{-} \mu^{+}$, measured as $\Gamma\left(K_{L} \rightarrow e \mu\right) / \Gamma\left(K_{L} \rightarrow\right.$ all $)<4.7 \times 10^{-12}$ and $\Gamma\left(K^{+} \rightarrow \pi^{+} e^{-} \mu^{+}\right) / \Gamma\left(K^{+} \rightarrow\right.$ all $)<1.3 \times 10^{-11}$. Limits on the conversion of $\tau$ into $e$ or $\mu$ are found in $\tau$ decay and are much less stringent than those for $\mu \rightarrow e$ conversion, e.g., $\Gamma(\tau \rightarrow \mu \gamma) / \Gamma(\tau \rightarrow$ all $)<6.8 \times 10^{-8}$ and $\Gamma(\tau \rightarrow e \gamma) / \Gamma(\tau \rightarrow$ all $)<1.1 \times 10^{-7}$.

c) Conversion of one type of charged lepton into another type of charged antilepton. The case most studied is $\mu^{-}+(Z, A) \rightarrow$ $e^{+}+(Z-2, A)$, the strongest limit being $\Gamma\left(\mu^{-} \mathrm{Ti} \rightarrow e^{+} \mathrm{Ca}\right) / \Gamma\left(\mu^{-} \mathrm{Ti} \rightarrow\right.$ all $)<3.6 \times 10^{-11}$.

d) Neutrino oscillations. If neutrinos have mass, then it is expected even in the standard electroweak theory that the lepton numbers are not separately conserved, as a consequence of lepton mixing analogous to Cabibbo quark mixing. However, if the only source of lepton-number violation is the mixing of low-mass neutrinos then processes such as $\mu \rightarrow e \gamma$ are expected to have extremely small unobservable probabilities. For small neutrino masses, the lepton-number violation would be observed first in neutrino oscillations, which have been the subject of extensive experimental searches. Strong evidence for neutrino mixing has come from atmospheric and solar neutrinos. The SNO experiment has detected the total flux of 
neutrinos from the sun measured via neutral current interactions and found it greater than the flux of $\nu_{e}$. This confirms previous indications of a deficit of $\nu_{e}$ and can be explained by oscillations with $\Delta\left(m^{2}\right)=\left(8.0_{-0.3}^{+0.4}\right) \times 10^{-5} \mathrm{eV}^{2}$. Evidence for such oscillations for reactor $\bar{\nu}$ has been found by the KAMLAND detector. In addition, underground detectors observing neutrinos produced by cosmic rays in the atmosphere have found a factor of 2 deficiency of upward going $\nu_{\mu}$ compared to downward. This provides compelling evidence for $\nu_{\mu}$ disappearance, for which the most probable explanation is $\nu_{\mu} \rightarrow \nu_{\tau}$ oscillations with nearly maximal mixing and $\Delta\left(\mathrm{m}^{2}\right)$ of the order 0.0019 $0.0030 \mathrm{eV}^{2}$.

\section{CONSERVATION OF HADRONIC FLAVORS}

In strong and electromagnetic interactions, hadronic flavor is conserved, i.e. the conversion of a quark of one flavor $(d, u, s, c, b, t)$ into a quark of another flavor is forbidden. In the Standard Model, the weak interactions violate these conservation laws in a manner described by the Cabibbo-Kobayashi-Maskawa mixing (see the section "Cabibbo-Kobayashi-Maskawa Mixing Matrix"). The way in which these conservation laws are violated is tested as follows:

(a) $\Delta S=\Delta Q$ rule. In the strangeness-changing semileptonic decay of strange particles, the strangeness change equals the change in charge of the hadrons. Tests come from limits on decay rates such as $\Gamma\left(\Sigma^{+} \rightarrow n e^{+} \nu\right) / \Gamma\left(\Sigma^{+} \rightarrow\right.$ all $)<5 \times 10^{-6}$, and from a detailed analysis of $K_{L} \rightarrow \pi e \nu$, which yields the parameter $x$, measured to be $(\operatorname{Re} x$, $\operatorname{Im} x)=(-0.002 \pm 0.006,0.0012 \pm 0.0021)$. Corresponding rules are $\Delta C=$ $\Delta Q$ and $\Delta B=\Delta Q$.

(b) Change of flavor by two units. In the Standard Model this occurs only in second-order weak interactions. The classic example is $\Delta S=$ 2 via $K^{0}-\bar{K}^{0}$ mixing, which is directly measured by $m\left(K_{L}\right)-m\left(K_{S}\right)=$ $(3.483 \pm 0.006) \times 10^{-12} \mathrm{MeV}$. There is now evidence for $B^{0}-\bar{B}^{0}$ mixing $(\Delta B=2)$, with the corresponding mass difference between the eigenstates 
$\left(m_{B_{\mathrm{H}}^{0}}-m_{B_{\mathrm{L}}^{0}}\right)=(0.776 \pm 0.008) \Gamma_{B^{0}}=(3.337 \pm 0.033) \times 10^{-10} \mathrm{MeV}$, and for $B_{s}^{0}-\bar{B}_{s}^{0}$ mixing, with $\left(m_{B_{s H}^{0}}-m_{B_{s L}^{0}}\right)>19.9 \Gamma_{B_{s}^{0}}$ or $>9 \times 10^{-9} \mathrm{MeV}$ $(\mathrm{CL}=95 \%)$. For $D^{0}-\bar{D}^{0}$ mixing $m_{D_{H}^{0}}-m_{D_{L}^{0}}<5 \times 10^{-11} \mathrm{MeV}$. All results are consistent with the second-order calculations in the Standard Model.

(c) Flavor-changing neutral currents. In the Standard Model the neutral-current interactions do not change flavor. The low rate $\Gamma\left(K_{L} \rightarrow\right.$ $\left.\mu^{+} \mu^{-}\right) / \Gamma\left(K_{L} \rightarrow\right.$ all $)=(6.87 \pm 0.11) \times 10^{-9}$ puts limits on such interactions; the nonzero value for this rate is attributed to a combination of the weak and electromagnetic interactions. The best test should come from $K^{+} \rightarrow \pi^{+} \nu \bar{\nu}$, which occurs in the Standard Model only as a second-order weak process with a branching fraction of $(0.4$ to 1.2$) \times 10^{-10}$. Recent results, including observation of two events, yields $\Gamma\left(K^{+} \rightarrow \pi^{+} \nu \bar{\nu}\right) / \Gamma\left(K^{+} \rightarrow\right.$ all $)$ $=\left(1.5_{-1.9}^{+1.3}\right) \times 10^{-10}[4]$. Limits for charm-changing or bottom-changing neutral currents are much less stringent: $\Gamma\left(D^{0} \rightarrow \mu^{+} \mu^{-}\right) / \Gamma\left(D^{0} \rightarrow\right.$ all $)<1.3 \times 10^{-6}$ and $\Gamma\left(B^{0} \rightarrow \mu^{+} \mu^{-}\right) / \Gamma\left(B^{0} \rightarrow\right.$ all $)<3.9 \times 10^{-8}$. One cannot isolate flavorchanging neutral current (FCNC) effects in non leptonic decays. For example, the FCNC transition $s \rightarrow d+(\bar{u}+u)$ is equivalent to the charged-current transition $s \rightarrow u+(\bar{u}+d)$. Tests for FCNC are therefore limited to hadron decays into lepton pairs. Such decays are expected only in second-order in the electroweak coupling in the Standard Model.

\section{References}

1. R. Carosi et al., Phys. Lett. B237, 303 (1990).

2. A. Alavi-Harati et al., Phys. Rev. D67, 012005 (2003);

B. Schwingenheuer et al., Phys. Rev. Lett. 74, 4376 (1995).

3. A. Angelopoulos et al., Phys. Lett. B444, 43 (1998);

L. Wolfenstein, Phys. Rev. Lett. 83, 911 (1999).

4. V.V. Animovsky et al., Phys. Rev. Lett. 93, 031801 (2004). 


\section{TESTS OF DISCRETE SPACE-TIME SYMMETRIES}

\section{CHARGE CONJUGATION (C) INVARIANCE}

$\Gamma\left(\pi^{0} \rightarrow 3 \gamma\right) / \Gamma_{\text {total }}$

$\Gamma\left(\pi^{0} \pi^{0} \pi^{0} \gamma\right) / \Gamma_{\text {total }}$

$\eta \mathrm{C}$-nonconserving decay parameters

$\pi^{+} \pi^{-} \pi^{0}$ left-right asymmetry parameter

$\pi^{+} \pi^{-} \pi^{0}$ sextant asymmetry parameter

$\pi^{+} \pi^{-} \pi^{0}$ quadrant asymmetry parameter

$\pi^{+} \pi^{-} \gamma$ left-right asymmetry parameter

$\pi^{+} \pi^{-} \gamma$ parameter $\beta$ ( $D$-wave)

$\Gamma\left(\eta \rightarrow \pi^{0} \gamma\right) / \Gamma_{\text {total }}$

$\Gamma\left(\eta \rightarrow \pi^{0} \pi^{0} \gamma\right) / \Gamma_{\text {total }}$

$\Gamma\left(\eta \rightarrow \pi^{0} \pi^{0} \pi^{0} \gamma\right) / \Gamma_{\text {total }}$

$\Gamma(\eta \rightarrow 3 \gamma) / \Gamma_{\text {total }}$

$\Gamma\left(\eta \rightarrow \pi^{0} e^{+} e^{-}\right) / \Gamma_{\text {total }}$

$\Gamma\left(\eta \rightarrow \pi^{0} \mu^{+} \mu^{-}\right) / \Gamma_{\text {total }}$

$\Gamma\left(\omega(782) \rightarrow \eta \pi^{0}\right) / \Gamma_{\text {total }}$

$\Gamma\left(\omega(782) \rightarrow 3 \pi^{0}\right) / \Gamma_{\text {total }}$

$\Gamma\left(\eta^{\prime}(958) \rightarrow \pi^{0} e^{+} e^{-}\right) / \Gamma_{\text {total }}$

$\Gamma\left(\eta^{\prime}(958) \rightarrow \eta e^{+} e^{-}\right) / \Gamma_{\text {total }}$

$\Gamma\left(\eta^{\prime}(958) \rightarrow 3 \gamma\right) / \Gamma_{\text {total }}$

$\Gamma\left(\eta^{\prime}(958) \rightarrow \mu^{+} \mu^{-} \pi^{0}\right) / \Gamma_{\text {total }}$

$\Gamma\left(\eta^{\prime}(958) \rightarrow \mu^{+} \mu^{-} \eta\right) / \Gamma_{\text {total }}$
$<3.1 \times 10^{-8}, \mathrm{CL}=90 \%$

$<6 \times 10^{-5}, \mathrm{CL}=90 \%$

$(0.09 \pm 0.17) \times 10^{-2}$

$(0.18 \pm 0.16) \times 10^{-2}$

$(-0.17 \pm 0.17) \times 10^{-2}$

$(0.9 \pm 0.4) \times 10^{-2}$

$-0.02 \pm 0.07(\mathrm{~S}=1.3)$

$<9 \times 10^{-5}, \mathrm{CL}=90 \%$

$<5 \times 10^{-4}, \mathrm{CL}=90 \%$

$<6 \times 10^{-5}, \mathrm{CL}=90 \%$

$<1.6 \times 10^{-5}, \mathrm{CL}=90 \%$

[a] $<4 \times 10^{-5}, \mathrm{CL}=90 \%$

[a] $<5 \times 10^{-6}, \mathrm{CL}=90 \%$

$<1 \times 10^{-3}, \mathrm{CL}=90 \%$

$<3 \times 10^{-4}, \mathrm{CL}=90 \%$

[a] $<1.4 \times 10^{-3}, \mathrm{CL}=90 \%$

[a] $<2.4 \times 10^{-3}, \mathrm{CL}=90 \%$

$<1.0 \times 10^{-4}, \mathrm{CL}=90 \%$

[a] $<6.0 \times 10^{-5}, \mathrm{CL}=90 \%$

[a] $<1.5 \times 10^{-5}, \mathrm{CL}=90 \%$

PARITY $(P)$ INVARIANCE

$e$ electric dipole moment

$\mu$ electric dipole moment

$\operatorname{Re}\left(d_{\tau}\right)$

$\Gamma\left(\eta \rightarrow \pi^{+} \pi^{-}\right) / \Gamma_{\text {total }}$

$\Gamma\left(\eta \rightarrow \pi^{0} \pi^{0}\right) / \Gamma_{\text {total }}$

$\Gamma\left(\eta \rightarrow 4 \pi^{0}\right) / \Gamma_{\text {total }}$

$\Gamma\left(\eta^{\prime}(958) \rightarrow \pi^{+} \pi^{-}\right) / \Gamma_{\text {total }}$

$\Gamma\left(\eta^{\prime}(958) \rightarrow \pi^{0} \pi^{0}\right) / \Gamma_{\text {total }}$

$\Gamma\left(\eta_{C}(1 S) \rightarrow \pi^{+} \pi^{-}\right) / \Gamma_{\text {total }}$

$\Gamma\left(\eta_{C}(1 S) \rightarrow \pi^{0} \pi^{0}\right) / \Gamma_{\text {total }}$

$\Gamma\left(\eta_{C}(1 S) \rightarrow K^{+} K^{-}\right) / \Gamma_{\text {total }}$

$\Gamma\left(\eta_{C}(1 S) \rightarrow K_{S}^{0} K_{S}^{0}\right) / \Gamma_{\text {total }}$

HTTP://PDG.LBL.GOV
$(0.07 \pm 0.07) \times 10^{-26} \mathrm{ecm}$

$(3.7 \pm 3.4) \times 10^{-19} \mathrm{ecm}$

-0.22 to $0.45 \times 10^{-16}$ e cm, CL $=95 \%$

$<1.3 \times 10^{-5}, \mathrm{CL}=90 \%$

$<4.3 \times 10^{-4}, \mathrm{CL}=90 \%$

$<6.9 \times 10^{-7}, \mathrm{CL}=90 \%$

$<2 \times 10^{-2}, \mathrm{CL}=90 \%$

$<9 \times 10^{-4}, \mathrm{CL}=90 \%$

$<8.7 \times 10^{-4}, \mathrm{CL}=90 \%$

$<5.6 \times 10^{-4}, \mathrm{CL}=90 \%$

$<7.6 \times 10^{-4}, \mathrm{CL}=90 \%$

$<4.2 \times 10^{-4}, \mathrm{CL}=90 \%$ 
$p$ electric dipole moment $n$ electric dipole moment $\Lambda$ electric dipole moment

$$
\begin{aligned}
& <0.54 \times 10^{-23} \text { e cm } \\
& <0.63 \times 10^{-25} \text { e cm, CL }=90 \% \\
& <1.5 \times 10^{-16} \text { e cm, CL }=95 \%
\end{aligned}
$$

\section{TIME REVERSAL $(T)$ INVARIANCE}

Limits on $e, \mu, \tau, p, n$, and $\Lambda$ electric dipole moments under Parity Invariance above are also tests of Time Reversal Invariance.

$\mu$ decay parameters

transverse $e^{+}$polarization normal to plane of $\mu$ spin, $e^{+}$momentum

$\alpha^{\prime} / A$

$\beta^{\prime} / A$

$P_{T}$ in $K^{+} \rightarrow \pi^{0} \mu^{+} \nu_{\mu}$

$P_{T}$ in $K^{+} \rightarrow \mu^{+} \nu_{\mu} \gamma$

$\operatorname{Im}(\xi)$ in $K^{+} \rightarrow \pi^{0} \mu^{+} \nu_{\mu}$ decay (from transverse $\mu$ pol.)

asymmetry $A_{T}$ in $K^{0}-\bar{K}^{0}$ mixing

$\operatorname{Im}(\xi)$ in $K_{\mu 3}^{0}$ decay (from transverse $\mu$ pol.)

$A_{T}\left(K_{S}^{0} K^{ \pm} \pi^{+} \pi^{-}\right)$in $D^{ \pm}$

$A_{T}\left(K^{+} K^{-} \pi^{+} \pi^{-}\right)$in $D^{0}, \bar{D}^{0}$

$A_{T}\left(K_{S}^{0} K^{ \pm} \pi^{+} \pi^{-}\right)$in $D_{S}^{ \pm}$

$n \rightarrow p e^{-} \bar{\nu}_{e}$ decay parameters

$\phi_{A V}$, phase of $g_{A}$ relative to $g_{V}$

triple correlation coefficient $D$

triple correlation coefficient $D$ for $\Sigma^{-} \rightarrow n e^{-} \bar{\nu}_{e}$
$(-2 \pm 8) \times 10^{-3}$

$(0 \pm 4) \times 10^{-3}$

$(1 \pm 5) \times 10^{-3}$

$(-1.7 \pm 2.5) \times 10^{-3}$

$(-0.6 \pm 1.9) \times 10^{-2}$

$-0.006 \pm 0.008$

$(6.6 \pm 1.6) \times 10^{-3}$

$-0.007 \pm 0.026$

$0.02 \pm 0.07$

$0.01 \pm 0.07$

$-0.04 \pm 0.07$

[b] $(180.06 \pm 0.07)^{\circ}$

$(-4 \pm 6) \times 10^{-4}$

$0.11 \pm 0.10$ 


\section{CP INVARIANCE}

$$
\begin{aligned}
& \operatorname{Re}\left(d_{\tau}^{w}\right) \\
& \operatorname{Im}\left(d_{\tau}^{W}\right) \\
& \Gamma\left(\eta \rightarrow \pi^{+} \pi^{-}\right) / \Gamma_{\text {total }} \\
& \Gamma\left(\eta \rightarrow \pi^{0} \pi^{0}\right) / \Gamma_{\text {total }} \\
& \Gamma\left(\eta \rightarrow 4 \pi^{0}\right) / \Gamma_{\text {total }} \\
& \Gamma\left(\eta^{\prime}(958) \rightarrow \pi^{+} \pi^{-}\right) / \Gamma_{\text {total }} \\
& \Gamma\left(\eta^{\prime}(958) \rightarrow \pi^{0} \pi^{0}\right) / \Gamma_{\text {total }} \\
& K^{ \pm} \rightarrow \pi^{ \pm} \pi^{+} \pi^{-} \text {rate difference/average } \\
& K^{ \pm} \rightarrow \pi^{ \pm} \pi^{0} \pi^{0} \text { rate difference/average } \\
& K^{ \pm} \rightarrow \pi^{ \pm} \pi^{0} \gamma \text { rate difference/average } \\
& K^{ \pm} \rightarrow \pi^{ \pm} \pi^{+} \pi^{-}\left(g_{+}-g_{-}\right) /\left(g_{+}+g_{-}\right) \\
& K^{ \pm} \rightarrow \pi^{ \pm} \pi^{0} \pi^{0}\left(g_{+}-g_{-}\right) /\left(g_{+}+g_{-}\right) \\
& \Delta\left(K_{\pi \mu \mu}^{ \pm}\right)=\frac{\Gamma\left(K_{\pi \mu \mu}^{+}\right)-\Gamma\left(K_{\pi \mu \mu}^{-}\right)}{\Gamma\left(K_{\pi \mu \mu}^{+}\right)+\Gamma\left(K_{\pi \mu \mu}^{-}\right)} \\
& \begin{aligned}
A_{S}= & {\left[\Gamma\left(K_{S}^{0} \rightarrow \pi^{-} e^{+} \nu_{e}\right)-\Gamma\left(K_{S}^{0} \rightarrow \pi^{+} e^{-} \bar{\nu}_{e}\right)\right] } \\
/ \operatorname{SUM} &
\end{aligned} \\
& \operatorname{Im}\left(\eta_{+-0}\right)=\operatorname{Im}\left(\mathrm{A}\left(K_{S}^{0} \rightarrow \pi^{+} \pi^{-} \pi^{0}, C P \text {-violating }\right)\right. \\
& \left./ \mathrm{A}\left(K_{L}^{0} \rightarrow \pi^{+} \pi^{-} \pi^{0}\right)\right) \\
& \operatorname{Im}\left(\eta_{000}\right)=\operatorname{Im}\left(A\left(K_{S}^{0} \rightarrow \pi^{0} \pi^{0} \pi^{0}\right) / A\left(K_{L}^{0} \rightarrow\right.\right. \\
& \left.\left.\pi^{0} \pi^{0} \pi^{0}\right)\right) \\
& \left|\eta_{000}\right|=\left|A\left(K_{S}^{0} \rightarrow 3 \pi^{0}\right) / A\left(K_{L}^{0} \rightarrow 3 \pi^{0}\right)\right| \\
& C P \text { asymmetry } A \text { in } K_{S}^{0} \rightarrow \pi^{+} \pi^{-} e^{+} e^{-} \\
& \Gamma\left(K_{S}^{0} \rightarrow 3 \pi^{0}\right) / \Gamma_{\text {total }} \\
& \text { linear coefficient } j \text { for } K_{L}^{0} \rightarrow \pi^{+} \pi^{-} \pi^{0} \\
& \text { quadratic coefficient } f \text { for } K_{L}^{0} \rightarrow \pi^{+} \pi^{-} \pi^{0} \\
& \left|\epsilon_{+-\gamma}^{\prime}\right| / \epsilon \text { for } K_{L}^{0} \rightarrow \pi^{+} \pi^{-} \gamma \\
& \Gamma\left(K_{L}^{0} \rightarrow \pi^{0} \mu^{+} \mu^{-}\right) / \Gamma_{\text {total }} \\
& \Gamma\left(K_{L}^{0} \rightarrow \pi^{0} e^{+} e^{-}\right) / \Gamma_{\text {total }} \\
& \Gamma\left(K_{L}^{0} \rightarrow \pi^{0} \nu \bar{\nu}\right) / \Gamma_{\text {total }} \\
& A_{C P}\left(K_{S}^{0} \pi^{ \pm}\right) \text {in } D^{ \pm} \\
& A_{C P}\left(K_{S}^{0} K^{ \pm}\right) \text {in } D^{ \pm} \\
& A_{C P}\left(K^{+} K^{-} \pi^{ \pm}\right) \text {in } D^{ \pm} \\
& A_{C P}\left(K^{ \pm} K^{* 0}\right) \text { in } D^{ \pm} \\
& A_{C P}\left(\phi \pi^{ \pm}\right) \text {in } D^{ \pm} \\
& A_{C P}\left(\pi^{+} \pi^{-} \pi^{ \pm}\right) \text {in } D^{ \pm} \\
& { }^{A} C P\left(K_{S}^{0} K^{ \pm} \pi^{+} \pi^{-}\right) \text {in } D^{ \pm} \\
& A_{C P}\left(K^{+} K^{-}\right) \text {in } D^{0}, \bar{D}^{0} \\
& { }^{A} C P\left(K_{S}^{0} K_{S}^{0}\right) \text { in } D^{0}, \bar{D}^{0} \\
& <0.50 \times 10^{-17} \text { e cm, CL }=95 \% \\
& <1.1 \times 10^{-17} \text { e cm, CL }=95 \% \\
& <1.3 \times 10^{-5}, \mathrm{CL}=90 \% \\
& <4.3 \times 10^{-4}, \mathrm{CL}=90 \% \\
& <6.9 \times 10^{-7}, \mathrm{CL}=90 \% \\
& <2 \times 10^{-2}, \mathrm{CL}=90 \% \\
& <9 \times 10^{-4}, \mathrm{CL}=90 \% \\
& (0.08 \pm 0.12) \% \\
& (0.0 \pm 0.6) \% \\
& (0.9 \pm 3.3) \% \\
& (1.5 \pm 2.9) \times 10^{-4} \\
& (0.02 \pm 0.19) \% \\
& -0.02 \pm 0.12 \\
& (2 \pm 10) \times 10^{-3} \\
& -0.002 \pm 0.009 \\
& (-0.1 \pm 1.6) \times 10^{-2} \\
& <0.018, \mathrm{CL}=90 \% \\
& (-1 \pm 4) \% \\
& <1.2 \times 10^{-7}, \mathrm{CL}=90 \% \\
& 0.0012 \pm 0.0008 \\
& 0.004 \pm 0.006 \\
& <0.3, \mathrm{CL}=90 \% \\
& \text { [c] }<3.8 \times 10^{-10}, \mathrm{CL}=90 \% \\
& \text { [c] }<2.8 \times 10^{-10}, \mathrm{CL}=90 \% \\
& \text { [d] }<5.9 \times 10^{-7}, \mathrm{CL}=90 \% \\
& -0.016 \pm 0.017 \\
& 0.07 \pm 0.06 \\
& 0.007 \pm 0.008 \\
& 0.005 \pm 0.017 \\
& -0.001 \pm 0.015 \\
& -0.02 \pm 0.04 \\
& -0.04 \pm 0.07 \\
& 0.014 \pm 0.010 \\
& -0.23 \pm 0.19
\end{aligned}
$$

HTTP://PDG.LBL.GOV 


\begin{tabular}{|c|c|}
\hline$A_{C P}\left(\pi^{+} \pi^{-}\right)$in $D^{0}, \bar{D}^{0}$ & $0.013 \pm 0.012$ \\
\hline$A_{C P}\left(\pi^{0} \pi^{0}\right)$ in $D^{0}, \bar{D}^{0}$ & $0.00 \pm 0.05$ \\
\hline${ }^{A} C P\left(\pi^{+} \pi^{-} \pi^{0}\right)$ in $D^{0}, \bar{D}^{0}$ & $0.01_{-0.09}^{+0.10}$ \\
\hline$A_{C P}\left(K_{S}^{0} \phi\right)$ in $D^{0}, \bar{D}^{0}$ & $-0.03 \pm 0.09$ \\
\hline$A_{C P}\left(K_{S}^{0} \pi^{0}\right)$ in $D^{0}, \bar{D}^{0}$ & $0.001 \pm 0.013$ \\
\hline$A_{C P}\left(K^{ \pm} \pi^{\mp}\right)$ in $D^{0}, \bar{D}^{0}$ & $0.05 \pm 0.04$ \\
\hline$A_{C P}\left(K^{\mp} \pi^{ \pm} \pi^{0}\right)$ in $D^{0}, \bar{D}^{0}$ & $-0.03 \pm 0.09$ \\
\hline$A_{C P}\left(K^{ \pm} \pi^{\mp} \pi^{0}\right)$ in $D^{0}, \bar{D}^{0}$ & $0.00 \pm 0.05$ \\
\hline$A_{C P}\left(K_{S}^{0} \pi^{+} \pi^{-}\right)$in $D^{0}, \bar{D}^{0}$ & $-0.009_{-0.061}^{+0.026}$ \\
\hline$A_{C P}\left(K^{ \pm} \pi^{\mp} \pi^{+} \pi^{-}\right)$in $D^{0}, \bar{D}^{0}$ & $-0.02 \pm 0.04$ \\
\hline$A_{C P}\left(K^{+} K^{-} \pi^{+} \pi^{-}\right)$in $D^{0}, \bar{D}^{0}$ & $-0.08 \pm 0.07$ \\
\hline$A_{C P}\left(B^{+} \rightarrow J / \psi(1 S) K^{+}\right)$ & $-0.024 \pm 0.014$ \\
\hline$A_{C P}\left(B^{+} \rightarrow J / \psi(1 S) \pi^{+}\right)$ & $0.09 \pm 0.08$ \\
\hline$A_{C P}\left(B^{+} \rightarrow J / \psi K^{*}(892)^{+}\right)$ & $0.048 \pm 0.033$ \\
\hline$A_{C P}\left(B^{+} \rightarrow \psi(2 S) K^{+}\right)$ & $-0.025 \pm 0.024$ \\
\hline$A_{C P}\left(B^{+} \rightarrow \psi(2 S) K^{*}(892)^{+}\right)$ & $-0.08 \pm 0.21$ \\
\hline$A_{C P}\left(B^{+} \rightarrow \chi_{C 1} K^{+}\right)$ & $0.00 \pm 0.08$ \\
\hline$A_{C P}\left(B^{+} \rightarrow \chi_{C 1} K^{*}(892)^{+}\right)$ & $-0.5 \pm 0.5$ \\
\hline$A_{C P}\left(B^{+} \rightarrow \bar{D}^{0} \pi^{+}\right)$ & $-0.008 \pm 0.008$ \\
\hline$A_{C P}\left(B^{+} \rightarrow D_{C P(+1)} \pi^{+}\right)$ & $0.035 \pm 0.024$ \\
\hline$A_{C P}\left(B^{+} \rightarrow D_{C P(-1)} \pi^{+}\right)$ & $0.017 \pm 0.026$ \\
\hline${ }^{A} C P\left(B^{+} \rightarrow \bar{D}^{0} K^{+}\right)$ & $0.07 \pm 0.04$ \\
\hline$A_{C P}\left(B^{+} \rightarrow\left[K^{-} \pi^{+}\right]_{D} K^{+}\right)$ & $0.9_{-0.6}^{+0.8}$ \\
\hline${ }^{A} C P\left(B^{+} \rightarrow\left[K^{-} \pi^{+}\right]_{\bar{D}} K^{*}(892)^{+}\right)$ & $-0.2 \pm 0.6$ \\
\hline$A_{C P}\left(B^{+} \rightarrow\left[K^{-} \pi^{+}\right]_{D} \pi^{+}\right)$ & $0.30_{-0.26}^{+0.30}$ \\
\hline$A_{C P}\left(B^{+} \rightarrow\left[\pi^{+} \pi^{-} \pi^{0}\right]_{D} K^{+}\right)$ & $-0.02 \pm 0.16$ \\
\hline$A_{C P}\left(B^{+} \rightarrow D_{C P(+1)} K^{+}\right)$ & $0.22 \pm 0.14(\mathrm{~S}=1.4)$ \\
\hline$A_{C P}\left(B^{+} \rightarrow D_{C P(-1)} K^{+}\right)$ & $-0.09 \pm 0.10$ \\
\hline$A_{C P}\left(B^{+} \rightarrow \bar{D}^{* 0} \pi^{+}\right)$ & $-0.014 \pm 0.015$ \\
\hline${ }^{A} C P\left(B^{+} \rightarrow\left(D_{C P(+1)}^{*}\right)^{0} \pi^{+}\right)$ & $-0.02 \pm 0.05$ \\
\hline$A_{C P}\left(B^{+} \rightarrow\left(D_{C P(-1)}^{*}\right)^{0} \pi^{+}\right)$ & $-0.09 \pm 0.05$ \\
\hline$A_{C P}\left(B^{+} \rightarrow D^{* 0} K^{+}\right)$ & $-0.09 \pm 0.09$ \\
\hline${ }^{A} C P\left(B^{+} \rightarrow D_{C P(+1)}^{* 0} K^{+}\right)$ & $-0.15 \pm 0.16$ \\
\hline$A_{C P}\left(B^{+} \rightarrow D_{C P(-1)}^{*} K^{+}\right)$ & $0.13 \pm 0.31$ \\
\hline$A_{C P}\left(B^{+} \rightarrow D_{C P(+1)} K^{*}(892)^{+}\right)$ & $-0.08 \pm 0.21$ \\
\hline$A_{C P}\left(B^{+} \rightarrow D_{C P(-1)} K^{*}(892)^{+}\right)$ & $-0.3 \pm 0.4$ \\
\hline$A_{C P}\left(B^{+} \rightarrow K_{S}^{0} \pi^{+}\right)$ & $-0.02 \pm 0.07(S=1.9)$ \\
\hline$A_{C P}\left(B^{+} \rightarrow K^{+} \pi^{0}\right)$ & $0.04 \pm 0.04$ \\
\hline
\end{tabular}

HTTP://PDG.LBL.GOV 


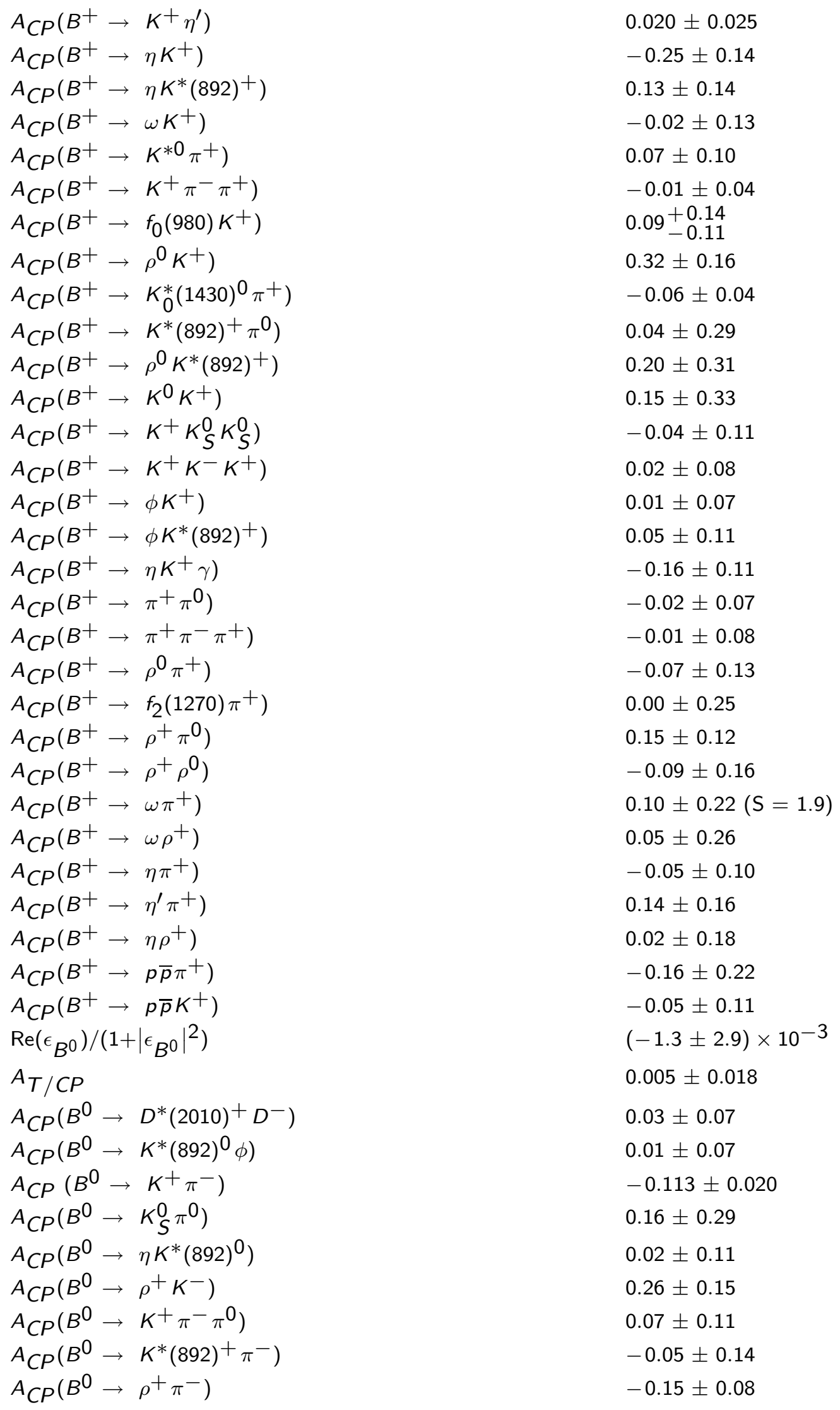

HTTP://PDG.LBL.GOV 


$$
\begin{aligned}
& A_{C P}\left(B^{0} \rightarrow \rho^{-} \pi^{+}\right) \\
& -0.53 \pm 0.30 \\
& A_{C P}\left(B^{0} \rightarrow K^{*}(1430) \gamma\right) \\
& -0.08 \pm 0.15 \\
& C_{D^{*}(2010)^{-} D^{+}}\left(B^{0} \rightarrow D^{*}(2010)^{-} D^{+}\right) \\
& 0.20 \pm 0.18 \\
& S_{D^{*}(2010)^{-} D^{+}}\left(B^{0} \rightarrow D^{*}(2010)^{-} D^{+}\right) \\
& -0.53 \pm 0.32(S=1.2) \\
& C_{D^{*}(2010)^{+} D^{-}}\left(B^{0} \rightarrow D^{*}(2010)^{+} D^{-}\right) \\
& -0.17 \pm 0.23(\mathrm{~S}=1.3) \\
& S_{D^{*}(2010)^{+} D^{-}}\left(B^{0} \rightarrow D^{*}(2010)^{+} D^{-}\right) \\
& -0.54 \pm 0.27 \\
& C_{D^{*+} D^{*-}}\left(B^{0} \rightarrow D^{*+} D^{*-}\right) \\
& 0.27 \pm 0.17 \\
& S_{D^{*+}} D^{*-}\left(B^{0} \rightarrow D^{*+} D^{*-}\right) \\
& -0.2 \pm 0.4(\mathrm{~S}=1.2) \\
& C_{+}\left(B^{0} \rightarrow D^{*+} D^{*-}\right) \\
& S_{+}\left(B^{0} \rightarrow D^{*+} D^{*-}\right) \\
& 0.06 \pm 0.17 \\
& C_{-}\left(B^{0} \rightarrow D^{*+} D^{*-}\right) \\
& -0.75 \pm 0.25 \\
& -0.2 \pm 1.0 \\
& S_{-}\left(B^{0} \rightarrow D^{*+} D^{*-}\right) \\
& C_{D^{+} D^{-}}\left(B^{0} \rightarrow D^{+} D^{-}\right) \\
& -1.8 \pm 1.8 \\
& S_{D^{+} D^{-}}\left(B^{0} \rightarrow D^{+} D^{-}\right) \\
& 0.1 \pm 0.4 \\
& C_{J / \psi(1 S) \pi^{0}}\left(B^{0} \rightarrow J / \psi(1 S) \pi^{0}\right) \\
& -0.3 \pm 0.6 \\
& S_{J / \psi(1 S) \pi^{0}}\left(B^{0} \rightarrow J / \psi(1 S) \pi^{0}\right) \\
& C_{\omega K_{S}^{0}}\left(B^{0} \rightarrow \omega K_{S}^{0}\right) \\
& S_{\omega K_{S}^{0}}\left(B^{0} \rightarrow \omega K_{S}^{0}\right) \\
& 0.13 \pm 0.24 \\
& -0.4 \pm 0.4(\mathrm{~S}=1.1) \\
& -0.3 \pm 0.5 \\
& 0.8 \pm 0.7 \\
& C_{\eta^{\prime}(958) K}\left(B^{0} \rightarrow \eta^{\prime}(958) K_{S}^{0}\right) \\
& -0.04 \pm 0.20(S=2.5) \\
& S_{\eta^{\prime}(958) K}\left(B^{0} \rightarrow \eta^{\prime}(958) K_{S}^{0}\right) \\
& 0.43 \pm 0.17(\mathrm{~S}=1.5) \\
& C_{f_{0}(980) K_{S}^{0}}\left(B^{0} \rightarrow f_{0}(980) K_{S}^{0}\right) \\
& 0.39 \pm 0.28 \\
& S_{f_{0}(980) K_{S}^{0}}\left(B^{0} \rightarrow f_{0}(980) K_{S}^{0}\right) \\
& 0.5 \pm 0.4 \\
& C_{K_{S}} K_{S} K_{S}\left(B^{0} \rightarrow K_{S} K_{S} K_{S}\right) \\
& S_{K_{S}} K_{S} K_{S}\left(B^{0} \rightarrow K_{S} K_{S} K_{S}\right) \\
& C_{K^{+} K^{-} K_{S}^{0}}\left(B^{0} \rightarrow K^{+} K^{-} K_{S}^{0}\right) \\
& -0.41 \pm 0.21 \\
& -0.3_{-0.7}^{+0.8}(\mathrm{~S}=2.4) \\
& 0.09 \pm 0.10 \\
& S_{K^{+} K^{-} K_{S}^{0}}\left(B^{0} \rightarrow K^{+} K^{-} K_{S}^{0}\right) \\
& -0.45 \pm 0.13 \\
& C_{\phi K_{S}^{0}}\left(B^{0} \rightarrow \phi K_{S}^{0}\right) \\
& -0.04 \pm 0.17 \\
& S_{\phi K_{S}^{0}}\left(B^{0} \rightarrow \phi K_{S}^{0}\right) \\
& 0.35 \pm 0.21 \\
& C_{K_{S}^{0} \pi^{0}}\left(B^{0} \rightarrow K_{S}^{0} \pi^{0}\right) \\
& 0.08 \pm 0.14 \\
& S_{K_{S}^{0} \pi^{0}}\left(B^{0} \rightarrow K_{S}^{0} \pi^{0}\right) \\
& 0.34 \pm 0.28 \\
& C_{K_{S}^{0} \pi^{0} \gamma}\left(B^{0} \rightarrow K_{S}^{0} \pi^{0} \gamma\right) \\
& -0.3 \pm 0.4(S=1.5) \\
& S_{K_{S}^{0} \pi^{0} \gamma}\left(B^{0} \rightarrow K_{S}^{0} \pi^{0} \gamma\right) \\
& -0.3_{-0.5}^{+0.6}(\mathrm{~S}=1.3) \\
& C_{K^{*}(892)^{0} \gamma}\left(B^{0} \rightarrow K^{*}(892)^{0} \gamma\right) \\
& -0.40 \pm 0.23
\end{aligned}
$$

HTTP://PDG.LBL.GOV 


$$
\begin{aligned}
& S_{K^{*}(892)^{0} \gamma}\left(B^{0} \rightarrow K^{*}(892)^{0} \gamma\right) \\
& C_{\pi \pi}\left(B^{0} \rightarrow \pi^{+} \pi^{-}\right) \\
& S_{\pi \pi}\left(B^{0} \rightarrow \pi^{+} \pi^{-}\right) \\
& C_{\pi^{0} \pi^{0}}\left(B^{0} \rightarrow \pi^{0} \pi^{0}\right) \\
& C_{\rho \pi}\left(B^{0} \rightarrow \rho^{+} \pi^{-}\right) \\
& S_{\rho \pi}\left(B^{0} \rightarrow \rho^{+} \pi^{-}\right) \\
& \Delta C_{\rho \pi}\left(B^{0} \rightarrow \rho^{+} \pi^{-}\right) \\
& \Delta S_{\rho \pi}\left(B^{0} \rightarrow \rho^{+} \pi^{-}\right) \\
& C_{\rho \rho}\left(B^{0} \rightarrow \rho^{+} \rho^{-}\right) \\
& S_{\rho \rho}\left(B^{0} \rightarrow \rho^{+} \rho^{-}\right) \\
& |\lambda|\left(B^{0} \rightarrow J / \psi K^{*}(892)^{0}\right) \\
& \left(\mathrm{S}_{+}+\mathrm{S}_{-}\right) / 2\left(B^{0} \rightarrow D^{*-} \pi^{+}\right) \\
& \left(\mathrm{S}_{-}-\mathrm{S}_{+}\right) / 2\left(B^{0} \rightarrow D^{*-} \pi^{+}\right) \\
& \left(\mathrm{S}_{+}+\mathrm{S}_{-}\right) / 2\left(B^{0} \rightarrow D^{-} \pi^{+}\right) \\
& \left(\mathrm{S}_{-}-\mathrm{S}_{+}\right) / 2\left(B^{0} \rightarrow D^{-} \pi^{+}\right) \\
& A_{C P}\left(B \rightarrow K^{*}(892) \gamma\right) \\
& A_{C P}(B \rightarrow s \gamma) \\
& A_{C P}\left(b \rightarrow X_{s} \ell^{+} \ell^{-}\right) \\
& \Gamma\left(\eta_{C}(1 S) \rightarrow \pi^{+} \pi^{-}\right) / \Gamma_{\text {total }} \\
& \Gamma\left(\eta_{C}(1 S) \rightarrow \pi^{0} \pi^{0}\right) / \Gamma_{\text {total }} \\
& \Gamma\left(\eta_{C}(1 S) \rightarrow K^{+} K^{-}\right) / \Gamma_{\text {total }} \\
& \Gamma\left(\eta_{C}(1 S) \rightarrow K_{S}^{0} K_{S}^{0}\right) / \Gamma_{\text {total }} \\
& {\left[\alpha_{-}(\Lambda)+\alpha_{+}(\bar{\Lambda})\right] /\left[\alpha_{-}(\Lambda)-\alpha_{+}(\bar{\Lambda})\right]} \\
& {\left[\alpha\left(\Xi^{-}\right) \alpha_{-}(\Lambda)-\alpha\left(\bar{\Xi}^{+}\right) \alpha_{+}(\bar{\Lambda})\right]} \\
& {\left[\alpha\left(\Xi^{-}\right) \alpha_{-}(\Lambda)+\alpha\left(\bar{\Xi}^{+}\right) \alpha_{+}(\bar{\Lambda})\right]} \\
& {\left[\alpha\left(\Omega^{-} \rightarrow \Lambda K^{-}\right)+\alpha\left(\bar{\Omega}^{+} \rightarrow \bar{\Lambda} K^{+}\right)\right] / 2} \\
& {\left[\alpha\left(\Lambda_{c}^{+}\right)+\alpha\left(\bar{\Lambda}_{c}^{-}\right)\right] /\left[\alpha\left(\Lambda_{c}^{+}\right)-\alpha\left(\bar{\Lambda}_{c}^{-}\right)\right] \text {in } \Lambda_{c}^{+} \rightarrow} \\
& \Lambda \pi^{+}, \bar{\Lambda}_{c}^{-} \rightarrow \bar{\Lambda} \pi^{-} \\
& {\left[\alpha\left(\Lambda_{c}^{+}\right)+\alpha\left(\bar{\Lambda}_{c}^{-}\right)\right] /\left[\alpha\left(\Lambda_{c}^{+}\right)-\alpha\left(\bar{\Lambda}_{c}^{-}\right)\right] \text {in } \Lambda_{c}^{+} \rightarrow} \\
& \Lambda e^{+} \nu_{e}, \bar{\Lambda}_{c}^{-} \rightarrow \bar{\Lambda} e^{-} \bar{\nu}_{e} \\
& -0.39 \pm 0.33 \\
& -0.36 \pm 0.23(\mathrm{~S}=2.3) \\
& -0.49 \pm 0.18(\mathrm{~S}=1.5) \\
& -0.3 \pm 0.4 \\
& 0.30 \pm 0.13 \\
& -0.04 \pm 0.23(\mathrm{~S}=1.3) \\
& 0.33 \pm 0.13 \\
& -0.07 \pm 0.22(\mathrm{~S}=1.3) \\
& -0.02 \pm 0.17 \\
& -0.22 \pm 0.22 \\
& <0.25, \mathrm{CL}=95 \% \\
& -0.028 \pm 0.017(\mathrm{~S}=1.3) \\
& -0.001 \pm 0.018 \\
& -0.043 \pm 0.030 \\
& -0.01 \pm 0.04 \\
& -0.010 \pm 0.028 \\
& 0.00 \pm 0.04 \\
& -0.22 \pm 0.26 \\
& <8.7 \times 10^{-4}, \mathrm{CL}=90 \% \\
& <5.6 \times 10^{-4}, \mathrm{CL}=90 \% \\
& <7.6 \times 10^{-4}, \mathrm{CL}=90 \% \\
& <4.2 \times 10^{-4}, \mathrm{CL}=90 \% \\
& 0.012 \pm 0.021 \\
& (0 \pm 7) \times 10^{-4} \\
& 0.00 \pm 0.04 \\
& -0.07 \pm 0.31
\end{aligned}
$$




\section{CP VIOLATION OBSERVED}

$\operatorname{Re}(\epsilon)$

charge asymmetry in $K_{\ell 3}^{0}$ decays

$A_{L}=$ weighted average of $A_{L}(\mu)$ and $A_{L}(e)$

$A_{L}(\mu)=\left[\Gamma\left(\pi^{-} \mu^{+} \nu_{\mu}\right)-\Gamma\left(\pi^{+} \mu^{-} \bar{\nu}_{\mu}\right)\right] /$ sum

$A_{L}(e)=\left[\Gamma\left(\pi^{-} e^{+} \nu_{e}\right)-\Gamma\left(\pi^{+} e^{-} \bar{\nu}_{e}\right)\right] /$ sum

parameters for $K_{L}^{0} \rightarrow 2 \pi$ decay

$$
\begin{aligned}
& \left|\eta_{00}\right|=\mid \mathrm{A}\left(K_{L}^{0} \rightarrow 2 \pi^{0}\right) / \\
& \quad \mathrm{A}\left(K_{S}^{0} \rightarrow 2 \pi^{0}\right) \mid \\
& \left|\eta_{+-}\right|=\mid \mathrm{A}\left(K_{L}^{0} \rightarrow \pi^{+} \pi^{-}\right) / \mathrm{A}\left(K_{S}^{0} \rightarrow\right. \\
& \left.\quad \pi^{+} \pi^{-}\right) \mid \\
& |\epsilon|=\left(2\left|\eta_{+-}\right|+\left|\eta_{00}\right|\right) / 3 \\
& \left|\eta_{00} / \eta_{+-}\right| \\
& \operatorname{Re}\left(\epsilon^{\prime} / \epsilon\right)=\left(1-\left|\eta_{00} / \eta_{+-}\right|\right) / 3
\end{aligned}
$$

Assuming $C P T$

$$
\begin{aligned}
& \phi_{+-}, \text {phase of } \eta_{+-} \\
& \phi_{00}, \text { phase of } \eta_{00} \\
& \phi_{\epsilon}=\left(2 \phi_{+-}+\phi_{00}\right) / 3
\end{aligned}
$$

Not assuming CPT

$$
\phi_{+-} \text {, phase of } \eta_{+-}
$$

$\phi_{00}$, phase of $\eta_{00}$

$$
\phi_{\epsilon}=\left(2 \phi_{+-}+\phi_{00}\right) / 3
$$

$C P$ asymmetry $A$ in $K_{L}^{0} \rightarrow \pi^{+} \pi^{-} e^{+} e^{-}$

$$
\beta_{C P} \text { from } K_{L}^{0} \rightarrow e^{+} e^{-} e^{+} e^{-}
$$$$
\gamma_{C P} \text { from } K_{L}^{0} \rightarrow e^{+} e^{-} e^{+} e^{-}
$$

parameters for $K_{L}^{0} \rightarrow \pi^{+} \pi^{-} \gamma$ decay

$$
\begin{array}{r}
\left|\eta_{+-\gamma}\right|=\mid \mathrm{A}\left(K_{L}^{0} \rightarrow \pi^{+} \pi^{-} \gamma, C P\right. \\
\text { violating }) / \mathrm{A}\left(K_{S}^{0} \rightarrow \pi^{+} \pi^{-} \gamma\right) \mid
\end{array}
$$

$$
\phi_{+-\gamma}=\text { phase of } \eta_{+-\gamma}
$$

$\Gamma\left(K_{L}^{0} \rightarrow \pi^{+} \pi^{-}\right) / \Gamma_{\text {total }}$

$\Gamma\left(K_{L}^{0} \rightarrow \pi^{0} \pi^{0}\right) / \Gamma_{\text {total }}$

$A_{C P}\left(B^{0} \rightarrow K^{+} \pi^{-}\right)$

Parameters for $B^{0} \rightarrow J / \psi K_{S}^{0}$

$$
\sin (2 \beta)
$$

$$
(1.664 \pm 0.010) \times 10^{-3}
$$

$$
\begin{aligned}
& (0.332 \pm 0.006) \% \\
& (0.304 \pm 0.025) \% \\
& (0.334 \pm 0.007) \%
\end{aligned}
$$

$$
(2.225 \pm 0.007) \times 10^{-3}
$$

$(2.236 \pm 0.007) \times 10^{-3}$

$$
(2.232 \pm 0.007) \times 10^{-3}
$$

[e] $0.9950 \pm 0.0008(\mathrm{~S}=1.6)$

[e] $(1.66 \pm 0.26) \times 10^{-3}(\mathrm{~S}=1.6)$

$$
\begin{aligned}
& (43.52 \pm 0.05)^{\circ}(S=1.2) \\
& (43.50 \pm 0.06)^{\circ}(S=1.2) \\
& (43.51 \pm 0.05)^{\circ}(S=1.1)
\end{aligned}
$$

$(43.4 \pm 0.7)^{\circ}(\mathrm{S}=1.3)$

$(43.7 \pm 0.8)^{\circ}(\mathrm{S}=1.2)$

$(43.5 \pm 0.7)^{\circ}(\mathrm{S}=1.3)$

$(13.7 \pm 1.5) \%$

$-0.19 \pm 0.07$

$0.01 \pm 0.11(S=1.6)$

$(2.35 \pm 0.07) \times 10^{-3}$

$(44 \pm 4)^{\circ}$

$(1.976 \pm 0.008) \times 10^{-3}$

$(8.69 \pm 0.04) \times 10^{-4}(\mathrm{~S}=1.1)$

$-0.113 \pm 0.020$

$0.725 \pm 0.037$ 


\section{CPT INVARIANCE}

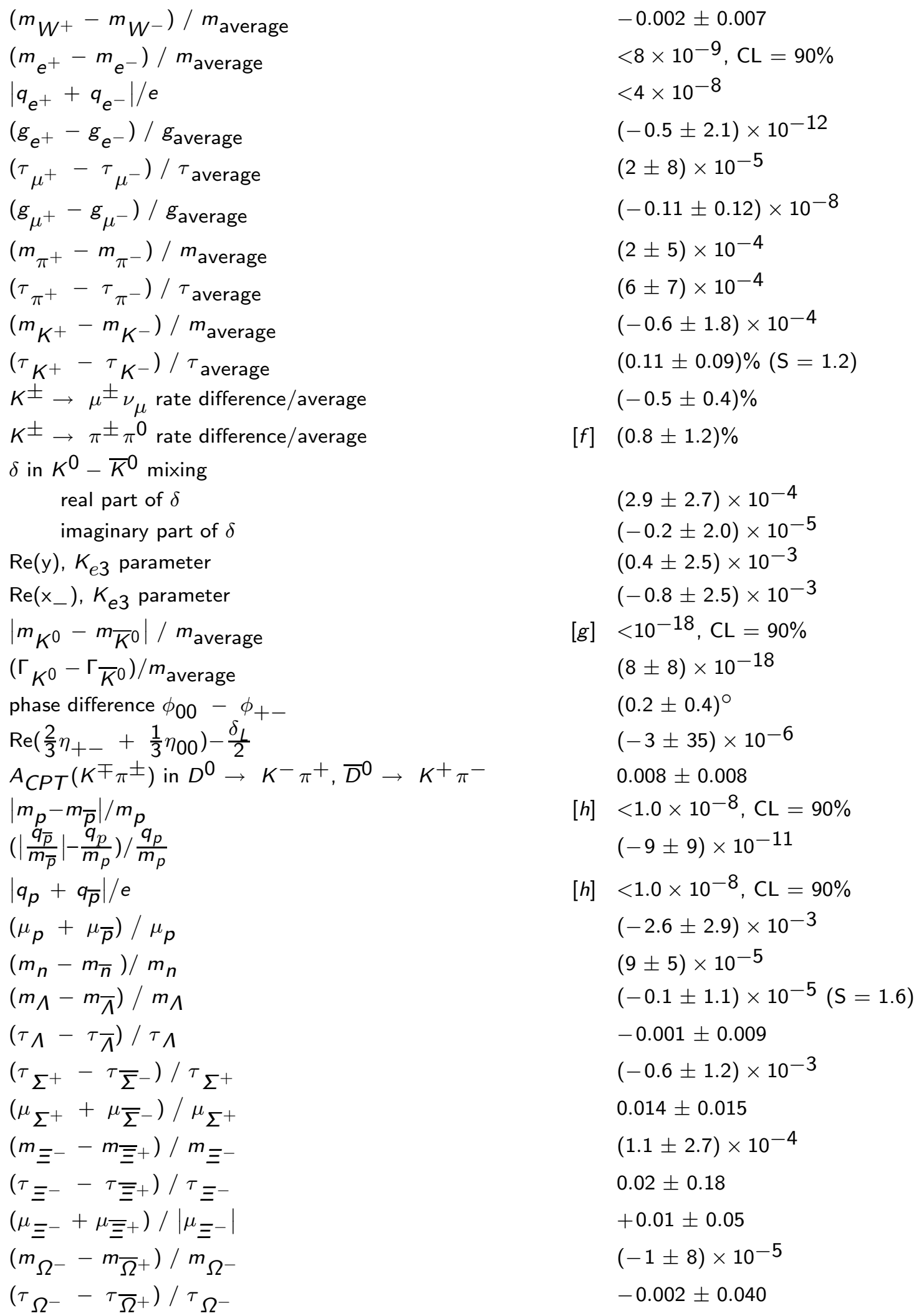




\section{TESTS OF NUMBER CONSERVATION LAWS}

\section{LEPTON FAMILY NUMBER}

Lepton family number conservation means separate conservation of each of $L_{e}, L_{\mu}$, $L_{\tau}$.

$$
\begin{aligned}
& \Gamma\left(Z \rightarrow e^{ \pm} \mu^{\mp}\right) / \Gamma_{\text {total }} \\
& \Gamma\left(Z \rightarrow e^{ \pm} \tau^{\mp}\right) / \Gamma_{\text {total }} \\
& \Gamma\left(Z \rightarrow \mu^{ \pm} \tau^{\mp}\right) / \Gamma_{\text {total }} \\
& \text { limit on } \mu^{-} \rightarrow e^{-} \text {conversion } \\
& \begin{array}{c}
\sigma\left(\mu^{-32} \mathrm{~S} \rightarrow e^{-32} \mathrm{~S}\right) / \\
\sigma\left(\mu^{-32} \mathrm{~S} \rightarrow \nu_{\mu}^{32} \mathrm{P}^{*}\right) \\
\sigma\left(\mu^{-} \mathrm{Ti} \rightarrow e^{-} \mathrm{Ti}\right) / \\
\sigma\left(\mu^{-} \mathrm{Ti} \rightarrow \text { capture }\right) \\
\sigma\left(\mu^{-} \mathrm{Pb} \rightarrow e^{-} \mathrm{Pb}\right) / \\
\sigma\left(\mu^{-} \mathrm{Pb} \rightarrow \text { capture }\right)
\end{array} \\
& G_{C} / G_{F} \\
& \Gamma\left(\mu^{-} \rightarrow e^{-} \nu_{e} \bar{\nu}_{\mu}\right) / \Gamma_{\text {total }} \\
& \Gamma\left(\mu^{-} \rightarrow e^{-} \gamma\right) / \Gamma_{\text {total }} \\
& \Gamma\left(\mu^{-} \rightarrow e^{-} e^{+} e^{-}\right) / \Gamma_{\text {total }} \\
& \Gamma\left(\mu^{-} \rightarrow e^{-} 2 \gamma\right) / \Gamma_{\text {total }} \\
& \Gamma\left(\tau^{-} \rightarrow e^{-} \gamma\right) / \Gamma_{\text {total }} \\
& \Gamma\left(\tau^{-} \rightarrow \mu^{-} \gamma\right) / \Gamma_{\text {total }} \\
& \Gamma\left(\tau^{-} \rightarrow e^{-} \pi^{0}\right) / \Gamma_{\text {total }} \\
& \Gamma\left(\tau^{-} \rightarrow \mu^{-} \pi^{0}\right) / \Gamma_{\text {total }} \\
& \Gamma\left(\tau^{-} \rightarrow e^{-} K_{S}^{0}\right) / \Gamma_{\text {total }} \\
& \Gamma\left(\tau^{-} \rightarrow \mu^{-} K_{S}^{0}\right) / \Gamma_{\text {total }} \\
& \Gamma\left(\tau^{-} \rightarrow e^{-} \eta\right) / \Gamma_{\text {total }} \\
& \Gamma\left(\tau^{-} \rightarrow \mu^{-} \eta\right) / \Gamma_{\text {total }} \\
& \Gamma\left(\tau^{-} \rightarrow e^{-} \rho^{0}\right) / \Gamma_{\text {total }} \\
& \Gamma\left(\tau^{-} \rightarrow \mu^{-} \rho^{0}\right) / \Gamma_{\text {total }} \\
& \Gamma\left(\tau^{-} \rightarrow e^{-} K^{*}(892)^{0}\right) / \Gamma_{\text {total }} \\
& \Gamma\left(\tau^{-} \rightarrow \mu^{-} K^{*}(892)^{0}\right) / \Gamma_{\text {total }} \\
& \Gamma\left(\tau^{-} \rightarrow e^{\left.-\bar{K}^{*}(892)^{0}\right) / \Gamma_{\text {total }}}\right. \\
& \Gamma\left(\tau^{-} \rightarrow \mu^{-} \bar{K}^{*}(892)^{0}\right) / \Gamma_{\text {total }} \\
& \Gamma\left(\tau^{-} \rightarrow e^{-} \eta^{\prime}(958)\right) / \Gamma_{\text {total }} \\
& \Gamma\left(\tau^{-} \rightarrow \mu^{-} \eta^{\prime}(958)\right) / \Gamma_{\text {total }} \\
& \Gamma\left(\tau^{-} \rightarrow e^{-} \phi\right) / \Gamma_{\text {total }} \\
& \Gamma\left(\tau^{-} \rightarrow \mu^{-} \phi\right) / \Gamma_{\text {total }}
\end{aligned}
$$$$
\text { limit on muonium } \rightarrow \text { antimuonium conversion } R_{g}=
$$

HTTP://PDG.LBL.GOV

$$
\begin{aligned}
& {[i]<1.7 \times 10^{-6}, \mathrm{CL}=95 \%} \\
& {[i]<9.8 \times 10^{-6}, \mathrm{CL}=95 \%} \\
& {[i]<1.2 \times 10^{-5}, \mathrm{CL}=95 \%}
\end{aligned}
$$$$
<7 \times 10^{-11}, \mathrm{CL}=90 \%
$$$$
<4.3 \times 10^{-12}, \mathrm{CL}=90 \%
$$$$
<4.6 \times 10^{-11}, \mathrm{CL}=90 \%
$$$$
<0.0030, \mathrm{CL}=90 \%
$$

[j] $<1.2 \times 10^{-2}, \mathrm{CL}=90 \%$

$<1.2 \times 10^{-11}, \mathrm{CL}=90 \%$

$<1.0 \times 10^{-12}, \mathrm{CL}=90 \%$

$<7.2 \times 10^{-11}, \mathrm{CL}=90 \%$

$<1.1 \times 10^{-7}, \mathrm{CL}=90 \%$

$<6.8 \times 10^{-8}, \mathrm{CL}=90 \%$

$<1.9 \times 10^{-7}, \mathrm{CL}=90 \%$

$<4.1 \times 10^{-7}, \mathrm{CL}=90 \%$

$<9.1 \times 10^{-7}, \mathrm{CL}=90 \%$

$<9.5 \times 10^{-7}, \mathrm{CL}=90 \%$

$<2.4 \times 10^{-7}, \mathrm{CL}=90 \%$

$<1.5 \times 10^{-7}, \mathrm{CL}=90 \%$

$<2.0 \times 10^{-6}, \mathrm{CL}=90 \%$

$<6.3 \times 10^{-6}, \mathrm{CL}=90 \%$

$<5.1 \times 10^{-6}, \mathrm{CL}=90 \%$

$<7.5 \times 10^{-6}, \mathrm{CL}=90 \%$

$<7.4 \times 10^{-6}, \mathrm{CL}=90 \%$

$<7.5 \times 10^{-6}, \mathrm{CL}=90 \%$

$<1.0 \times 10^{-6}, \mathrm{CL}=90 \%$

$<4.7 \times 10^{-7}, \mathrm{CL}=90 \%$

$<6.9 \times 10^{-6}, \mathrm{CL}=90 \%$

$<7.0 \times 10^{-6}, \mathrm{CL}=90 \%$

Page 15
Created: 5/1/2007 17:24 


$$
\begin{aligned}
& \Gamma\left(\tau^{-} \rightarrow e^{-} e^{+} e^{-}\right) / \Gamma_{\text {total }} \\
& \Gamma\left(\tau^{-} \rightarrow e^{-} \mu^{+} \mu^{-}\right) / \Gamma_{\text {total }} \\
& \Gamma\left(\tau^{-} \rightarrow e^{+} \mu^{-} \mu^{-}\right) / \Gamma_{\text {total }} \\
& \Gamma\left(\tau^{-} \rightarrow \mu^{-} e^{+} e^{-}\right) / \Gamma_{\text {total }} \\
& \Gamma\left(\tau^{-} \rightarrow \mu^{+} e^{-} e^{-}\right) / \Gamma_{\text {total }} \\
& \Gamma\left(\tau^{-} \rightarrow \mu^{-} \mu^{+} \mu^{-}\right) / \Gamma_{\text {total }} \\
& \Gamma\left(\tau^{-} \rightarrow e^{-} \pi^{+} \pi^{-}\right) / \Gamma_{\text {total }} \\
& \Gamma\left(\tau^{-} \rightarrow \mu^{-} \pi^{+} \pi^{-}\right) / \Gamma_{\text {total }} \\
& \Gamma\left(\tau^{-} \rightarrow e^{-} \pi^{+} K^{-}\right) / \Gamma_{\text {total }} \\
& \Gamma\left(\tau^{-} \rightarrow e^{-} \pi^{-} K^{+}\right) / \Gamma_{\text {total }} \\
& \Gamma\left(\tau^{-} \rightarrow e^{-} K_{S}^{0} K_{S}^{0}\right) / \Gamma_{\text {total }} \\
& \Gamma\left(\tau^{-} \rightarrow e^{-} K^{+} K^{-}\right) / \Gamma_{\text {total }} \\
& \Gamma\left(\tau^{-} \rightarrow \mu^{-} \pi^{+} K^{-}\right) / \Gamma_{\text {total }} \\
& \Gamma\left(\tau^{-} \rightarrow \mu^{-} \pi^{-} K^{+}\right) / \Gamma_{\text {total }} \\
& \Gamma\left(\tau^{-} \rightarrow \mu^{-} K_{S}^{0} K_{S}^{0}\right) / \Gamma_{\text {total }} \\
& \Gamma\left(\tau^{-} \rightarrow \mu^{-} K^{+} K^{-}\right) / \Gamma_{\text {total }} \\
& \Gamma\left(\tau^{-} \rightarrow e^{-} \pi^{0} \pi^{0}\right) / \Gamma_{\text {total }} \\
& \Gamma\left(\tau^{-} \rightarrow \mu^{-} \pi^{0} \pi^{0}\right) / \Gamma_{\text {total }} \\
& \Gamma\left(\tau^{-} \rightarrow e^{-} \eta \eta\right) / \Gamma_{\text {total }} \\
& \Gamma\left(\tau^{-} \rightarrow \mu^{-} \eta \eta\right) / \Gamma_{\text {total }} \\
& \Gamma\left(\tau^{-} \rightarrow e^{-} \pi^{0} \eta\right) / \Gamma_{\text {total }} \\
& \Gamma\left(\tau^{-} \rightarrow \mu^{-} \pi^{0} \eta\right) / \Gamma_{\text {total }} \\
& \Gamma\left(\tau^{-} \rightarrow e^{-} \text {light boson }\right) / \Gamma_{\text {total }} \\
& \Gamma\left(\tau^{-} \rightarrow \mu^{-} \text {light boson }\right) / \Gamma_{\text {total }}
\end{aligned}
$$

\section{LEPTON FAMILY NUMBER VIOLATION IN NEUTRINOS}

Solar Neutrinos

$$
\begin{aligned}
& \sin ^{2}\left(2 \theta_{12}\right) \\
& \Delta \mathrm{m}_{21}^{2}
\end{aligned}
$$

Atmospheric Neutrinos

$$
\begin{aligned}
& \sin ^{2}\left(2 \theta_{23}\right) \\
& \Delta \mathrm{m}_{32}^{2}
\end{aligned}
$$

$\Gamma\left(\pi^{+} \rightarrow \mu^{+} \nu_{e}\right) / \Gamma_{\text {total }}$

$\Gamma\left(\pi^{+} \rightarrow \mu^{-} e^{+} e^{+} \nu\right) / \Gamma_{\text {total }}$

$\Gamma\left(\pi^{0} \rightarrow \mu^{+} e^{-}\right) / \Gamma_{\text {total }}$

$\Gamma\left(\pi^{0} \rightarrow \mu^{-} e^{+}\right) / \Gamma_{\text {total }}$

$\Gamma\left(\pi^{0} \rightarrow \mu^{+} e^{-}+\mu^{-} e^{+}\right) / \Gamma_{\text {total }}$

$\Gamma\left(\eta \rightarrow \mu^{+} e^{-}+\mu^{-} e^{+}\right) / \Gamma_{\text {total }}$

$\Gamma\left(\eta^{\prime}(958) \rightarrow e \mu\right) / \Gamma_{\text {total }}$

$\Gamma\left(K^{+} \rightarrow \mu^{-} \nu e^{+} e^{+}\right) / \Gamma_{\text {total }}$

$\Gamma\left(K^{+} \rightarrow \mu^{+} \nu_{e}\right) / \Gamma_{\text {total }}$

$\Gamma\left(K^{+} \rightarrow \pi^{+} \mu^{+} e^{-}\right) / \Gamma_{\text {total }}$

$\Gamma\left(K^{+} \rightarrow \pi^{+} \mu^{-} e^{+}\right) / \Gamma_{\text {total }}$

HTTP://PDG.LBL.GOV

$$
\begin{aligned}
& <2.0 \times 10^{-7}, \mathrm{CL}=90 \% \\
& <2.0 \times 10^{-7}, \mathrm{CL}=90 \% \\
& <1.3 \times 10^{-7}, \mathrm{CL}=90 \% \\
& <1.9 \times 10^{-7}, \mathrm{CL}=90 \% \\
& <1.1 \times 10^{-7}, \mathrm{CL}=90 \% \\
& <1.9 \times 10^{-7}, \mathrm{CL}=90 \% \\
& <1.2 \times 10^{-7}, \mathrm{CL}=90 \% \\
& <2.9 \times 10^{-7}, \mathrm{CL}=90 \% \\
& <3.2 \times 10^{-7}, \mathrm{CL}=90 \% \\
& <1.7 \times 10^{-7}, \mathrm{CL}=90 \% \\
& <2.2 \times 10^{-6}, \mathrm{CL}=90 \% \\
& <1.4 \times 10^{-7}, \mathrm{CL}=90 \% \\
& <2.6 \times 10^{-7}, \mathrm{CL}=90 \% \\
& <3.2 \times 10^{-7}, \mathrm{CL}=90 \% \\
& <3.4 \times 10^{-6}, \mathrm{CL}=90 \% \\
& <2.5 \times 10^{-7}, \mathrm{CL}=90 \% \\
& <6.5 \times 10^{-6}, \mathrm{CL}=90 \% \\
& <1.4 \times 10^{-5}, \mathrm{CL}=90 \% \\
& <3.5 \times 10^{-5}, \mathrm{CL}=90 \% \\
& <6.0 \times 10^{-5}, \mathrm{CL}=90 \% \\
& <2.4 \times 10^{-5}, \mathrm{CL}=90 \% \\
& <2.2 \times 10^{-5}, \mathrm{CL}=90 \% \\
& <2.7 \times 10^{-3}, \mathrm{CL}=95 \% \\
& <5 \times 10^{-3}, \mathrm{CL}=95 \%
\end{aligned}
$$

$$
\begin{aligned}
& 0.86_{-0.04}^{+0.03} \\
& \left(8.0_{-0.3}^{+0.4}\right) \times 10^{-5} \mathrm{eV}^{2}
\end{aligned}
$$

$>0.92$

[k] 1.9 to $3.0 \times 10^{-3} \mathrm{eV}^{2}$

[l] $<8.0 \times 10^{-3}, \mathrm{CL}=90 \%$

$<1.6 \times 10^{-6}, \mathrm{CL}=90 \%$

$<3.8 \times 10^{-10}, \mathrm{CL}=90 \%$

$<3.4 \times 10^{-9}, \mathrm{CL}=90 \%$

$<1.72 \times 10^{-8}, \mathrm{CL}=90 \%$

$<6 \times 10^{-6}, \mathrm{CL}=90 \%$

$<4.7 \times 10^{-4}, \mathrm{CL}=90 \%$

$<2.0 \times 10^{-8}, \mathrm{CL}=90 \%$

[l] $<4 \times 10^{-3}, \mathrm{CL}=90 \%$

$<1.3 \times 10^{-11}, \mathrm{CL}=90 \%$

$<5.2 \times 10^{-10}, \mathrm{CL}=90 \%$

Page 16

Created: 5/1/2007 17:24 


$$
\begin{aligned}
& \Gamma\left(K_{L}^{0} \rightarrow e^{ \pm} \mu^{\mp}\right) / \Gamma_{\text {total }} \\
& \Gamma\left(K_{L}^{0} \rightarrow e^{ \pm} e^{ \pm} \mu^{\mp} \mu^{\mp}\right) / \Gamma_{\text {total }} \\
& \Gamma\left(K_{L}^{0} \rightarrow \pi^{0} \mu^{ \pm} e^{\mp}\right) / \Gamma_{\text {total }} \\
& \Gamma\left(D^{+} \rightarrow \pi^{+} e^{ \pm} \mu^{\mp}\right) / \Gamma_{\text {total }} \\
& \Gamma\left(D^{+} \rightarrow K^{+} e^{ \pm} \mu^{\mp}\right) / \Gamma_{\text {total }} \\
& \Gamma\left(D^{0} \rightarrow \mu^{ \pm} e^{\mp}\right) / \Gamma_{\text {total }} \\
& \Gamma\left(D^{0} \rightarrow \pi^{0} e^{ \pm} \mu^{\mp}\right) / \Gamma_{\text {total }} \\
& \Gamma\left(D^{0} \rightarrow \eta e^{ \pm} \mu^{\mp}\right) / \Gamma_{\text {total }} \\
& \Gamma\left(D^{0} \rightarrow \pi^{+} \pi^{-} e^{ \pm} \mu^{\mp}\right) / \Gamma_{\text {total }} \\
& \Gamma\left(D^{0} \rightarrow \rho^{0} e^{ \pm} \mu^{\mp}\right) / \Gamma_{\text {total }} \\
& \Gamma\left(D^{0} \rightarrow \omega e^{ \pm} \mu^{\mp}\right) / \Gamma_{\text {total }} \\
& \Gamma\left(D^{0} \rightarrow K^{-} K^{+} e^{ \pm} \mu^{\mp}\right) / \Gamma_{\text {total }} \\
& \Gamma\left(D^{0} \rightarrow \phi e^{ \pm} \mu^{\mp}\right) / \Gamma_{\text {total }} \\
& \Gamma\left(D^{0} \rightarrow \bar{K}^{0} e^{ \pm} \mu^{\mp}\right) / \Gamma_{\text {total }} \\
& \Gamma\left(D^{0} \rightarrow K^{-} \pi^{+} e^{ \pm} \mu^{\mp}\right) / \Gamma_{\text {total }} \\
& \Gamma\left(D^{0} \rightarrow \bar{K}^{*}(892)^{0} e^{ \pm} \mu^{\mp}\right) / \Gamma_{\text {total }} \\
& \Gamma\left(D_{s}^{+} \rightarrow \pi^{+} e^{ \pm} \mu^{\mp}\right) / \Gamma_{\text {total }} \\
& \Gamma\left(D_{s}^{+} \rightarrow K^{+} e^{ \pm} \mu^{\mp}\right) / \Gamma_{\text {total }} \\
& \Gamma\left(B^{+} \rightarrow \pi^{+} e^{+} \mu^{-}\right) / \Gamma_{\text {total }} \\
& \Gamma\left(B^{+} \rightarrow \pi^{+} e^{-} \mu^{+}\right) / \Gamma_{\text {total }} \\
& \Gamma\left(B^{+} \rightarrow K^{+} e^{+} \mu^{-}\right) / \Gamma_{\text {total }} \\
& \Gamma\left(B^{+} \rightarrow K^{+} e^{-} \mu^{+}\right) / \Gamma_{\text {total }} \\
& \Gamma\left(B^{+} \rightarrow K^{*}(892)^{+} e^{ \pm} \mu^{\mp}\right) / \Gamma_{\text {total }} \\
& \Gamma\left(B^{0} \rightarrow e^{ \pm} \mu^{\mp}\right) / \Gamma_{\text {total }} \\
& \Gamma\left(B^{0} \rightarrow K^{0} e^{ \pm} \mu^{\mp}\right) / \Gamma_{\text {total }} \\
& \Gamma\left(B^{0} \rightarrow K^{*}(892)^{0} e^{ \pm} \mu^{\mp}\right) / \Gamma_{\text {total }} \\
& \Gamma\left(B^{0} \rightarrow e^{ \pm} \tau^{\mp}\right) / \Gamma_{\text {total }} \\
& \Gamma\left(B^{0} \rightarrow \mu^{ \pm} \tau^{\mp}\right) / \Gamma_{\text {total }} \\
& \Gamma\left(B \rightarrow e^{ \pm} \mu^{\mp} s\right) / \Gamma_{\text {total }} \\
& \Gamma\left(B \rightarrow \pi e^{ \pm} \mu^{\mp}\right) / \Gamma_{\text {total }} \\
& \Gamma\left(B \rightarrow \rho e^{ \pm} \mu^{\mp}\right) / \Gamma_{\text {total }} \\
& \Gamma\left(B \rightarrow K e^{ \pm} \mu^{\mp}\right) / \Gamma_{\text {total }} \\
& \Gamma\left(B \rightarrow K^{*}(892) e^{ \pm} \mu^{\mp}\right) / \Gamma_{\text {total }} \\
& \Gamma\left(B_{s}^{0} \rightarrow e^{ \pm} \mu^{\mp}\right) / \Gamma_{\text {total }} \\
& \Gamma\left(e^{ \pm} \tau^{\mp}\right) / \Gamma_{\text {total }} \\
& \Gamma\left(\mu^{ \pm} \tau^{\mp}\right) / \Gamma_{\text {total }} \\
& \Gamma\left(J / \psi(1 S) \rightarrow e^{ \pm} \mu^{\mp}\right) / \Gamma_{\text {total }} \\
& \Gamma\left(J / \psi(1 S) \rightarrow e^{ \pm} \tau^{\mp}\right) / \Gamma_{\text {total }} \\
& \Gamma\left(J / \psi(1 S) \rightarrow \mu^{ \pm} \tau^{\mp}\right) / \Gamma_{\text {total }}
\end{aligned}
$$

[i] $<4.7 \times 10^{-12}, \mathrm{CL}=90 \%$

[i] $<4.12 \times 10^{-11}, \mathrm{CL}=90 \%$

[i] $<6.2 \times 10^{-9}, \mathrm{CL}=90 \%$

[i] $<3.4 \times 10^{-5}, \mathrm{CL}=90 \%$

[i] $<6.8 \times 10^{-5}, \mathrm{CL}=90 \%$

[i] $<8.1 \times 10^{-7}, \mathrm{CL}=90 \%$

[i] $<8.6 \times 10^{-5}, \mathrm{CL}=90 \%$

[i] $<1.0 \times 10^{-4}, \mathrm{CL}=90 \%$

[i] $<1.5 \times 10^{-5}, \mathrm{CL}=90 \%$

[i] $<4.9 \times 10^{-5}, \mathrm{CL}=90 \%$

[i] $<1.2 \times 10^{-4}, \mathrm{CL}=90 \%$

[i] $<1.8 \times 10^{-4}, \mathrm{CL}=90 \%$

[i] $<3.4 \times 10^{-5}, \mathrm{CL}=90 \%$

[i] $<1.0 \times 10^{-4}, \mathrm{CL}=90 \%$

[i] $<5.53 \times 10^{-4}, \mathrm{CL}=90 \%$

[i] $<8.3 \times 10^{-5}, \mathrm{CL}=90 \%$

[i] $<6.1 \times 10^{-4}, \mathrm{CL}=90 \%$

[i] $<6.3 \times 10^{-4}, \mathrm{CL}=90 \%$

$<6.4 \times 10^{-3}, \mathrm{CL}=90 \%$

$<6.4 \times 10^{-3}, \mathrm{CL}=90 \%$

$<8 \times 10^{-7}, \mathrm{CL}=90 \%$

$<6.4 \times 10^{-3}, \mathrm{CL}=90 \%$

$<7.9 \times 10^{-6}, \mathrm{CL}=90 \%$

[i] $<1.7 \times 10^{-7}, \mathrm{CL}=90 \%$

$<4.0 \times 10^{-6}, \mathrm{CL}=90 \%$

$<3.4 \times 10^{-6}, \mathrm{CL}=90 \%$

[i] $<1.1 \times 10^{-4}, \mathrm{CL}=90 \%$

[i] $<3.8 \times 10^{-5}, \mathrm{CL}=90 \%$

[i] $<2.2 \times 10^{-5}, \mathrm{CL}=90 \%$

$<1.6 \times 10^{-6}, \mathrm{CL}=90 \%$

$<3.2 \times 10^{-6}, \mathrm{CL}=90 \%$

$<1.6 \times 10^{-6}, \mathrm{CL}=90 \%$

$<6.2 \times 10^{-6}, \mathrm{CL}=90 \%$

[i] $<6.1 \times 10^{-6}, \mathrm{CL}=90 \%$

$<8.3 \times 10^{-6}, \mathrm{CL}=90 \%$

$<2.0 \times 10^{-6}, \mathrm{CL}=90 \%$

$<1.1 \times 10^{-6}, \mathrm{CL}=90 \%$

$<8.3 \times 10^{-6}, \mathrm{CL}=90 \%$

$<2.0 \times 10^{-6}, \mathrm{CL}=90 \%$ 


\section{TOTAL LEPTON NUMBER}

Violation of total lepton number conservation also implies violation of lepton family number conservation.

$$
\begin{aligned}
& \Gamma(Z \rightarrow p e) / \Gamma_{\text {total }} \\
& \Gamma(Z \rightarrow p \mu) / \Gamma_{\text {total }} \\
& \text { limit on } \mu^{-} \rightarrow e^{+} \text {conversion } \\
& \sigma\left(\mu^{-32} \mathrm{~S} \rightarrow e^{+32} \mathrm{Si}^{*}\right) / \\
& \sigma\left(\mu^{-32} \mathrm{~S} \rightarrow \nu_{\mu}{ }^{32} \mathrm{P}^{*}\right) \\
& \sigma\left(\mu^{-127} \mathrm{I} \rightarrow e^{+127} \mathrm{Sb}^{*}\right) / \\
& \sigma\left(\mu^{-127} \mathrm{I} \rightarrow \text { anything }\right) \\
& \sigma\left(\mu^{-} \mathrm{Ti} \rightarrow e^{+} \mathrm{Ca}\right) / \\
& \sigma\left(\mu^{-} \mathrm{Ti} \rightarrow \text { capture }\right) \\
& \Gamma\left(\tau^{-} \rightarrow e^{+} \pi^{-} \pi^{-}\right) / \Gamma_{\text {total }} \\
& \Gamma\left(\tau^{-} \rightarrow \mu^{+} \pi^{-} \pi^{-}\right) / \Gamma_{\text {total }} \\
& \Gamma\left(\tau^{-} \rightarrow e^{+} \pi^{-} K^{-}\right) / \Gamma_{\text {total }} \\
& \Gamma\left(\tau^{-} \rightarrow e^{+} K^{-} K^{-}\right) / \Gamma_{\text {total }} \\
& \Gamma\left(\tau^{-} \rightarrow \mu^{+} \pi^{-} K^{-}\right) / \Gamma_{\text {total }} \\
& \Gamma\left(\tau^{-} \rightarrow \mu^{+} K^{-} K^{-}\right) / \Gamma_{\text {total }} \\
& \Gamma\left(\tau^{-} \rightarrow \bar{p} \gamma\right) / \Gamma_{\text {total }} \\
& \Gamma\left(\tau^{-} \rightarrow \bar{p} \pi^{0}\right) / \Gamma_{\text {total }} \\
& \Gamma\left(\tau^{-} \rightarrow \bar{p} 2 \pi^{0}\right) / \Gamma_{\text {total }} \\
& \Gamma\left(\tau^{-} \rightarrow \bar{p} \eta\right) / \Gamma_{\text {total }} \\
& \Gamma\left(\tau^{-} \rightarrow \bar{p} \pi^{0} \eta\right) / \Gamma_{\text {total }} \\
& \Gamma\left(\tau^{-} \rightarrow \Lambda \pi^{-}\right) / \Gamma_{\text {total }} \\
& \Gamma\left(\tau^{-} \rightarrow \bar{\Lambda} \pi^{-}\right) / \Gamma_{\text {total }} \\
& t_{1 / 2}\left({ }^{76} \mathrm{Ge} \rightarrow{ }^{76} \mathrm{Se}+2 e^{-}\right) \\
& \Gamma\left(\pi^{+} \rightarrow \mu^{+} \bar{\nu}_{e}\right) / \Gamma_{\text {total }} \\
& \Gamma\left(K^{+} \rightarrow \pi^{-} \mu^{+} e^{+}\right) / \Gamma_{\text {total }} \\
& \Gamma\left(K^{+} \rightarrow \pi^{-} e^{+} e^{+}\right) / \Gamma_{\text {total }} \\
& \Gamma\left(K^{+} \rightarrow \pi^{-} \mu^{+} \mu^{+}\right) / \Gamma_{\text {total }} \\
& \Gamma\left(K^{+} \rightarrow \mu^{+} \bar{\nu}_{e}\right) / \Gamma_{\text {total }} \\
& \Gamma\left(K^{+} \rightarrow \pi^{0} e^{+} \bar{\nu}_{e}\right) / \Gamma_{\text {total }} \\
& \Gamma\left(D^{+} \rightarrow \pi^{-} e^{+} e^{+}\right) / \Gamma_{\text {total }} \\
& \Gamma\left(D^{+} \rightarrow \pi^{-} \mu^{+} \mu^{+}\right) / \Gamma_{\text {total }} \\
& \Gamma\left(D^{+} \rightarrow \pi^{-} e^{+} \mu^{+}\right) / \Gamma_{\text {total }} \\
& \Gamma\left(D^{+} \rightarrow \rho^{-} \mu^{+} \mu^{+}\right) / \Gamma_{\text {total }} \\
& \Gamma\left(D^{+} \rightarrow K^{-} e^{+} e^{+}\right) / \Gamma_{\text {total }} \\
& \Gamma\left(D^{+} \rightarrow K^{-} \mu^{+} \mu^{+}\right) / \Gamma_{\text {total }} \\
& \Gamma\left(D^{+} \rightarrow K^{-} e^{+} \mu^{+}\right) / \Gamma_{\text {total }} \\
& \Gamma\left(D^{+} \rightarrow K^{*}(892)^{-} \mu^{+} \mu^{+}\right) / \Gamma_{\text {total }} \\
& \Gamma\left(D^{0} \rightarrow \pi^{-} \pi^{-} e^{+} e^{+}+\text {c.c. }\right) / \Gamma_{\text {total }}
\end{aligned}
$$

HTTP://PDG.LBL.GOV

$$
\begin{aligned}
& <1.8 \times 10^{-6}, \mathrm{CL}=95 \% \\
& <1.8 \times 10^{-6}, \mathrm{CL}=95 \% \\
& <9 \times 10^{-10}, \mathrm{CL}=90 \% \\
& <3 \times 10^{-10}, \mathrm{CL}=90 \% \\
& <3.6 \times 10^{-11}, \mathrm{CL}=90 \% \\
& <2.7 \times 10^{-7}, \mathrm{CL}=90 \% \\
& <7 \times 10^{-8}, \mathrm{CL}=90 \% \\
& <1.8 \times 10^{-7}, \mathrm{CL}=90 \% \\
& <1.5 \times 10^{-7}, \mathrm{CL}=90 \% \\
& <2.2 \times 10^{-7}, \mathrm{CL}=90 \% \\
& <4.8 \times 10^{-7}, \mathrm{CL}=90 \% \\
& <3.5 \times 10^{-6}, \mathrm{CL}=90 \% \\
& <1.5 \times 10^{-5}, \mathrm{CL}=90 \% \\
& <3.3 \times 10^{-5}, \mathrm{CL}=90 \% \\
& <8.9 \times 10^{-6}, \mathrm{CL}=90 \% \\
& <2.7 \times 10^{-5}, \mathrm{CL}=90 \% \\
& <7.2 \times 10^{-8}, \mathrm{CL}=90 \% \\
& <1.4 \times 10^{-7}, \mathrm{CL}=90 \% \\
& >1.9 \times 10^{25} \mathrm{yr}, \mathrm{CL}=90 \% \\
& \text { [l] }<1.5 \times 10^{-3}, \mathrm{CL}=90 \% \\
& <5.0 \times 10^{-10}, \mathrm{CL}=90 \% \\
& <6.4 \times 10^{-10}, \mathrm{CL}=90 \% \\
& \text { [l] }<3.0 \times 10^{-9}, \mathrm{CL}=90 \% \\
& \text { [l] }<3.3 \times 10^{-3}, \mathrm{CL}=90 \% \\
& <3 \times 10^{-3}, \mathrm{CL}=90 \% \\
& <3.6 \times 10^{-6}, \mathrm{CL}=90 \% \\
& <4.8 \times 10^{-6}, \mathrm{CL}=90 \% \\
& <5.0 \times 10^{-5}, \mathrm{CL}=90 \% \\
& <5.6 \times 10^{-4}, \mathrm{CL}=90 \% \\
& <4.5 \times 10^{-6}, \mathrm{CL}=90 \% \\
& <1.3 \times 10^{-5}, \mathrm{CL}=90 \% \\
& <1.3 \times 10^{-4}, \mathrm{CL}=90 \% \\
& <8.5 \times 10^{-4}, \mathrm{CL}=90 \% \\
& <1.12 \times 10^{-4}, \mathrm{CL}=90 \%
\end{aligned}
$$

Page 18

Created: 5/1/2007 17:24 


$$
\begin{aligned}
& \Gamma\left(D^{0} \rightarrow \pi^{-} \pi^{-} \mu^{+} \mu^{+}+\text {c.c. }\right) / \Gamma_{\text {total }} \\
& \Gamma\left(D^{0} \rightarrow K^{-} \pi^{-} e^{+} e^{+}+\text {c.c. }\right) / \Gamma_{\text {total }} \\
& \Gamma\left(D^{0} \rightarrow K^{-} \pi^{-} \mu^{+} \mu^{+}+\text {c.c. }\right) / \Gamma_{\text {total }} \\
& \Gamma\left(D^{0} \rightarrow K^{-} K^{-} e^{+} e^{+}+\text {c.c. }\right) / \Gamma_{\text {total }} \\
& \Gamma\left(D^{0} \rightarrow K^{-} K^{-} \mu^{+} \mu^{+}+\text {c.c. }\right) / \Gamma_{\text {total }} \\
& \Gamma\left(D^{0} \rightarrow \pi^{-} \pi^{-} e^{+} \mu^{+}+\text {c.c. }\right) / \Gamma_{\text {total }} \\
& \Gamma\left(D^{0} \rightarrow K^{-} \pi^{-} e^{+} \mu^{+}+\text {c.c. }\right) / \Gamma_{\text {total }} \\
& \Gamma\left(D^{0} \rightarrow K^{-} K^{-} e^{+} \mu^{+}+\text {c.c. }\right) / \Gamma_{\text {total }} \\
& \Gamma\left(D_{s}^{+} \rightarrow \pi^{-} e^{+} e^{+}\right) / \Gamma_{\text {total }} \\
& \Gamma\left(D_{s}^{+} \rightarrow \pi^{-} \mu^{+} \mu^{+}\right) / \Gamma_{\text {total }} \\
& \Gamma\left(D_{s}^{+} \rightarrow \pi^{-} e^{+} \mu^{+}\right) / \Gamma_{\text {total }} \\
& \Gamma\left(D_{s}^{+} \rightarrow K^{-} e^{+} e^{+}\right) / \Gamma_{\text {total }} \\
& \Gamma\left(D_{s}^{+} \rightarrow K^{-} \mu^{+} \mu^{+}\right) / \Gamma_{\text {total }} \\
& \Gamma\left(D_{s}^{+} \rightarrow K^{-} e^{+} \mu^{+}\right) / \Gamma_{\text {total }} \\
& \Gamma\left(D_{s}^{+} \rightarrow K^{*}(892)-\mu^{+} \mu^{+}\right) / \Gamma_{\text {total }} \\
& \Gamma\left(B^{+} \rightarrow \pi^{-} e^{+} e^{+}\right) / \Gamma_{\text {total }} \\
& \Gamma\left(B^{+} \rightarrow \pi^{-} \mu^{+} \mu^{+}\right) / \Gamma_{\text {total }} \\
& \Gamma\left(B^{+} \rightarrow \pi^{-} e^{+} \mu^{+}\right) / \Gamma_{\text {total }} \\
& \Gamma\left(B^{+} \rightarrow \rho^{-} e^{+} e^{+}\right) / \Gamma_{\text {total }} \\
& \Gamma\left(B^{+} \rightarrow \rho^{-} \mu^{+} \mu^{+}\right) / \Gamma_{\text {total }} \\
& \Gamma\left(B^{+} \rightarrow \rho^{-} e^{+} \mu^{+}\right) / \Gamma_{\text {total }} \\
& \Gamma\left(B^{+} \rightarrow K^{-} e^{+} e^{+}\right) / \Gamma_{\text {total }} \\
& \Gamma\left(B^{+} \rightarrow K^{-} \mu^{+} \mu^{+}\right) / \Gamma_{\text {total }} \\
& \Gamma\left(B^{+} \rightarrow K^{-} e^{+} \mu^{+}\right) / \Gamma_{\text {total }} \\
& \Gamma\left(B^{+} \rightarrow K^{*}(892)^{-} e^{+} e^{+}\right) / \Gamma_{\text {total }} \\
& \Gamma\left(B^{+} \rightarrow K^{*}(892)^{-} \mu^{+} \mu^{+}\right) / \Gamma_{\text {total }} \\
& \Gamma\left(B^{+} \rightarrow K^{*}(892)^{-} e^{+} \mu^{+}\right) / \Gamma_{\text {total }} \\
& \Gamma\left(\Xi^{-} \rightarrow \mu^{-} \mu^{-}\right) / \Gamma_{\text {total }} \\
& \Gamma\left(\Lambda_{c}^{+} \rightarrow \Sigma^{-} \mu^{+} \mu^{+}\right) / \Gamma_{\text {total }} \\
&
\end{aligned}
$$

$$
\begin{aligned}
& <2.9 \times 10^{-5}, \mathrm{CL}=90 \% \\
& <2.06 \times 10^{-4}, \mathrm{CL}=90 \% \\
& <3.9 \times 10^{-4}, \mathrm{CL}=90 \% \\
& <1.52 \times 10^{-4}, \mathrm{CL}=90 \% \\
& <9.4 \times 10^{-5}, \mathrm{CL}=90 \% \\
& <7.9 \times 10^{-5}, \mathrm{CL}=90 \% \\
& <2.18 \times 10^{-4}, \mathrm{CL}=90 \% \\
& <5.7 \times 10^{-5}, \mathrm{CL}=90 \% \\
& <6.9 \times 10^{-4}, \mathrm{CL}=90 \% \\
& <2.9 \times 10^{-5}, \mathrm{CL}=90 \% \\
& <7.3 \times 10^{-4}, \mathrm{CL}=90 \% \\
& <6.3 \times 10^{-4}, \mathrm{CL}=90 \% \\
& <1.3 \times 10^{-5}, \mathrm{CL}=90 \% \\
& <6.8 \times 10^{-4}, \mathrm{CL}=90 \% \\
& <1.4 \times 10^{-3}, \mathrm{CL}=90 \% \\
& <1.6 \times 10^{-6}, \mathrm{CL}=90 \% \\
& <1.4 \times 10^{-6}, \mathrm{CL}=90 \% \\
& <1.3 \times 10^{-6}, \mathrm{CL}=90 \% \\
& <2.6 \times 10^{-6}, \mathrm{CL}=90 \% \\
& <5.0 \times 10^{-6}, \mathrm{CL}=90 \% \\
& <3.3 \times 10^{-6}, \mathrm{CL}=90 \% \\
& <1.0 \times 10^{-6}, \mathrm{CL}=90 \% \\
& <1.8 \times 10^{-6}, \mathrm{CL}=90 \% \\
& <2.0 \times 10^{-6}, \mathrm{CL}=90 \% \\
& <2.8 \times 10^{-6}, \mathrm{CL}=90 \% \\
& <8.3 \times 10^{-6}, \mathrm{CL}=90 \% \\
& <4.4 \times 10^{-6}, \mathrm{CL}=90 \% \\
& <4 \times 10^{-8}, \mathrm{CL}=90 \% \\
& <7.0 \times 10^{-4}, \mathrm{CL}=90 \%
\end{aligned}
$$

Page 19

Created: 5/1/2007 17:24 


\section{BARYON NUMBER}

$\Gamma(Z \rightarrow p e) / \Gamma_{\text {total }}$

$\Gamma(Z \rightarrow p \mu) / \Gamma_{\text {total }}$

$\Gamma\left(\tau^{-} \rightarrow \bar{p} \gamma\right) / \Gamma_{\text {total }}$

$\Gamma\left(\tau^{-} \rightarrow \bar{p} \pi^{0}\right) / \Gamma_{\text {total }}$

$\Gamma\left(\tau^{-} \rightarrow \bar{p} 2 \pi^{0}\right) / \Gamma_{\text {total }}$

$\Gamma\left(\tau^{-} \rightarrow \bar{p} \eta\right) / \Gamma_{\text {total }}$

$\Gamma\left(\tau^{-} \rightarrow \bar{p} \pi^{0} \eta\right) / \Gamma_{\text {total }}$

$\Gamma\left(\tau^{-} \rightarrow \Lambda \pi^{-}\right) / \Gamma_{\text {total }}$

$\Gamma\left(\tau^{-} \rightarrow \bar{\Lambda} \pi^{-}\right) / \Gamma_{\text {total }}$

$p$ mean life

$$
\begin{aligned}
& <1.8 \times 10^{-6}, \mathrm{CL}=95 \% \\
& <1.8 \times 10^{-6}, \mathrm{CL}=95 \% \\
& <3.5 \times 10^{-6}, \mathrm{CL}=90 \% \\
& <1.5 \times 10^{-5}, \mathrm{CL}=90 \% \\
& <3.3 \times 10^{-5}, \mathrm{CL}=90 \% \\
& <8.9 \times 10^{-6}, \mathrm{CL}=90 \% \\
& <2.7 \times 10^{-5}, \mathrm{CL}=90 \% \\
& <7.2 \times 10^{-8}, \mathrm{CL}=90 \% \\
& <1.4 \times 10^{-7}, \mathrm{CL}=90 \% \\
& >1.9 \times 10^{29} \text { years, } \mathrm{CL}=90 \%
\end{aligned}
$$

A few examples of proton or bound neutron decay follow. For limits on many other nucleon decay channels, see the Baryon Summary Table.

$\tau\left(N \rightarrow e^{+} \pi\right)$

$\tau\left(N \rightarrow \mu^{+} \pi\right)$

$\tau\left(N \rightarrow e^{+} K\right)$

$\tau\left(N \rightarrow \mu^{+} K\right)$

limit on $n \bar{n}$ oscillations (free $n$ )

limit on $n \bar{n}$ oscillations (bound $n$ )

$$
\begin{gathered}
>158(n),>1600(p) \times 10^{30} \text { years, } \mathrm{CL}= \\
90 \% \\
>100(n),>473(p) \times 10^{30} \text { years, } \mathrm{CL}= \\
90 \% \\
>17(n),>150(p) \times 10^{30} \text { years, } \mathrm{CL}= \\
90 \% \\
>26(n),>120(p) \times 10^{30} \text { years, } \mathrm{CL}= \\
90 \% \\
>0.86 \times 10^{8} \mathrm{~s}, \mathrm{CL}=90 \% \\
{[m]>1.2 \times 10^{8} \mathrm{~s}, \mathrm{CL}=90 \%}
\end{gathered}
$$

\section{ELECTRIC CHARGE (Q)}

$e \rightarrow \nu_{e} \gamma$ and astrophysical limits

$\Gamma\left(n \rightarrow p \nu_{e} \bar{\nu}_{e}\right) / \Gamma_{\text {total }}$

$$
\begin{aligned}
{[n] } & >4.6 \times 10^{26} \mathrm{yr}, \mathrm{CL}=90 \% \\
& <8 \times 10^{-27}, \mathrm{CL}=68 \%
\end{aligned}
$$




\section{$\Delta S=\Delta Q R U L E$}

Violations allowed in second-order weak interactions.

$$
\begin{array}{ll}
\Gamma\left(K^{+} \rightarrow \pi^{+} \pi^{+} e^{-} \bar{\nu}_{e}\right) / \Gamma_{\text {total }} & <1.2 \times 10^{-8}, \mathrm{CL}=90 \% \\
\Gamma\left(K^{+} \rightarrow \pi^{+} \pi^{+} \mu^{-} \bar{\nu}_{\mu}\right) / \Gamma_{\text {total }} & <3.0 \times 10^{-6}, \mathrm{CL}=95 \% \\
\quad \operatorname{Re}\left(\mathrm{x}_{+}\right), K_{e 3} \text { parameter } & (-0.8 \pm 3.1) \times 10^{-3} \\
x=\mathrm{A}\left(\bar{K}^{0} \rightarrow \pi^{-} \ell^{+} \nu\right) / \mathrm{A}\left(K^{0} \rightarrow \pi^{-} \ell^{+} \nu\right)=\mathrm{A}(\Delta S=-\Delta Q) / \mathrm{A}(\Delta S=\Delta Q) & -0.002 \pm 0.006 \\
\quad \quad \quad \text { imal part of } x & 0.0012 \pm 0.0021 \\
\quad \Gamma\left(\Sigma^{+} \rightarrow n \ell^{+} \nu\right) / \Gamma\left(\Sigma^{-} \rightarrow n \ell^{-} \bar{\nu}\right) & <0.043 \\
\Gamma\left(\Sigma^{+} \rightarrow n e^{+} \nu_{e}\right) / \Gamma_{\text {total }} & <5 \times 10^{-6}, \mathrm{CL}=90 \% \\
\Gamma\left(\Sigma^{+} \rightarrow n \mu^{+} \nu_{\mu}\right) / \Gamma_{\text {total }} & <3.0 \times 10^{-5}, \mathrm{CL}=90 \% \\
\Gamma\left(\Xi^{0} \rightarrow \Sigma^{-} e^{+} \nu_{e}\right) / \Gamma_{\text {total }} & <9 \times 10^{-4}, \mathrm{CL}=90 \% \\
\Gamma\left(\Xi^{0} \rightarrow \Sigma^{-} \mu^{+} \nu_{\mu}\right) / \Gamma_{\text {total }} & <9 \times 10^{-4}, \mathrm{CL}=90 \%
\end{array}
$$

\section{$\Delta S=2$ FORBIDDEN}

Allowed in second-order weak interactions.

$$
\begin{aligned}
& \Gamma\left(\Xi^{0} \rightarrow p \pi^{-}\right) / \Gamma_{\text {total }} \\
& \Gamma\left(\Xi^{0} \rightarrow p e^{-} \bar{\nu}_{e}\right) / \Gamma_{\text {total }} \\
& \Gamma\left(\Xi^{0} \rightarrow p \mu^{-} \bar{\nu}_{\mu}\right) / \Gamma_{\text {total }} \\
& \Gamma\left(\Xi^{-} \rightarrow n \pi^{-}\right) / \Gamma_{\text {total }} \\
& \Gamma\left(\Xi^{-} \rightarrow n e^{-} \bar{\nu}_{e}\right) / \Gamma_{\text {total }} \\
& \Gamma\left(\Xi^{-} \rightarrow n \mu^{-} \bar{\nu}_{\mu}\right) / \Gamma_{\text {total }} \\
& \Gamma\left(\Xi^{-} \rightarrow p \pi^{-} \pi^{-}\right) / \Gamma_{\text {total }} \\
& \Gamma\left(\Xi^{-} \rightarrow p \pi^{-} e^{-} \bar{\nu}_{e}\right) / \Gamma_{\text {total }} \\
& \Gamma\left(\Xi^{-} \rightarrow p \pi^{-} \mu^{-} \bar{\nu}_{\mu}\right) / \Gamma_{\text {total }} \\
& \Gamma\left(\Omega^{-} \rightarrow \Lambda \pi^{-}\right) / \Gamma_{\text {total }}
\end{aligned}
$$

$$
\begin{aligned}
& <8 \times 10^{-6}, \mathrm{CL}=90 \% \\
& <1.3 \times 10^{-3} \\
& <1.3 \times 10^{-3} \\
& <1.9 \times 10^{-5}, \mathrm{CL}=90 \% \\
& <3.2 \times 10^{-3}, \mathrm{CL}=90 \% \\
& <1.5 \times 10^{-2}, \mathrm{CL}=90 \% \\
& <4 \times 10^{-4}, \mathrm{CL}=90 \% \\
& <4 \times 10^{-4}, \mathrm{CL}=90 \% \\
& <4 \times 10^{-4}, \mathrm{CL}=90 \% \\
& <2.9 \times 10^{-6}, \mathrm{CL}=90 \%
\end{aligned}
$$

\section{$\Delta S=2$ VIA MIXING}

Allowed in second-order weak interactions, e.g. mixing.
$m_{K_{L}^{0}}-m_{K_{S}^{0}}$
$(0.5292 \pm 0.0009) \times 10^{10} \hbar \mathrm{s}^{-1}(\mathrm{~S}=1.2)$
$m_{K_{L}^{0}}-m_{K_{S}^{0}}$
$(3.483 \pm 0.006) \times 10^{-12} \mathrm{MeV}$

HTTP://PDG.LBL.GOV 


\section{$\Delta C=2$ VIA MIXING}

Allowed in second-order weak interactions, e.g. mixing.

$$
\begin{aligned}
& \left|m_{D_{1}^{0}}-m_{D_{2}^{0}}\right| \\
& \left(\Gamma_{D_{1}^{0}}-\Gamma_{D_{2}^{0}}\right) / \Gamma=2 y \\
& \Gamma\left(D^{0} \rightarrow K^{+} \ell^{-} \bar{\nu}_{\ell}\left(\text { via } \bar{D}^{0}\right)\right) / \Gamma_{\text {total }} \\
& \Gamma\left(D^{0} \rightarrow K^{+} \text {or } K^{*}(892)^{+} e^{-} \bar{\nu}_{e}\left(\text { via } \bar{D}^{0}\right)\right) / \Gamma_{\text {total }} \\
& \Gamma\left(D^{0} \rightarrow K^{+} \pi^{-}\left(\operatorname{via}^{0}\right)\right) / \Gamma_{\text {total }} \\
& \Gamma\left(D^{0} \rightarrow K_{S}^{0} \pi^{+} \pi^{-}\left(\text {in } D^{0} \rightarrow \bar{D}^{0}\right)\right) / \Gamma_{\text {total }} \\
& \Gamma\left(D^{0} \rightarrow K^{+} \pi^{-} \pi^{+} \pi^{-}\left(\text {via } \bar{D}^{0}\right)\right) / \Gamma_{\text {total }} \\
& \Gamma\left(D^{0} \rightarrow \mu^{-} \text {anything }\left(\text { via } \bar{D}^{0}\right)\right) / \Gamma_{\text {total }}
\end{aligned}
$$

$$
\begin{aligned}
\text { [o] } & <7 \times 10^{10} \hbar \mathrm{s}^{-1}, \mathrm{CL}=95 \% \\
& (1.4 \pm 1.0) \times 10^{-4} \\
& <1.8 \times 10^{-4}, \mathrm{CL}=90 \% \\
& <6 \times 10^{-5}, \mathrm{CL}=90 \% \\
& <1.5 \times 10^{-5}, \mathrm{CL}=95 \% \\
& <1.8 \times 10^{-4}, \mathrm{CL}=95 \% \\
& <4 \times 10^{-4}, \mathrm{CL}=90 \% \\
& <4 \times 10^{-4}, \mathrm{CL}=90 \%
\end{aligned}
$$

\section{$\Delta B=2$ VIA MIXING}

Allowed in second-order weak interactions, e.g. mixing.

$$
\begin{aligned}
& \chi_{d} \\
& \Delta m_{B^{0}}=m_{B_{H}^{0}}-m_{B_{L}^{0}} \\
& x_{d}=\Delta m_{B^{0}} / \Gamma_{B^{0}} \\
& \Delta m_{B_{s}^{0}}=m_{B_{s H}^{0}}-m_{B_{s L}^{0}} \\
& x_{s}=\Delta m_{B_{s}^{0}} / \Gamma_{B_{s}^{0}} \\
& \chi_{s}
\end{aligned}
$$$$
0.188 \pm 0.003
$$$$
(0.507 \pm 0.005) \times 10^{12} \hbar \mathrm{s}^{-1}
$$$$
0.776 \pm 0.008
$$$$
>14.4 \times 10^{12} \hbar \mathrm{s}^{-1}, \mathrm{CL}=95 \%
$$$$
>19.9, \mathrm{CL}=95 \%
$$$$
>0.49878, C L=95 \%
$$

\section{$\Delta S=1$ WEAK NEUTRAL CURRENT FORBIDDEN}

Allowed by higher-order electroweak interactions.

$$
\begin{aligned}
& \Gamma\left(K^{+} \rightarrow \pi^{+} e^{+} e^{-}\right) / \Gamma_{\text {total }} \\
& \Gamma\left(K^{+} \rightarrow \pi^{+} \mu^{+} \mu^{-}\right) / \Gamma_{\text {total }} \\
& \Gamma\left(K^{+} \rightarrow \pi^{+} \nu \bar{\nu}\right) / \Gamma_{\text {total }} \\
& \Gamma\left(K^{+} \rightarrow \pi^{+} \pi^{0} \nu \bar{\nu}\right) / \Gamma_{\text {total }} \\
& \Gamma\left(K_{S}^{0} \rightarrow \mu^{+} \mu^{-}\right) / \Gamma_{\text {total }} \\
& \Gamma\left(K_{S}^{0} \rightarrow e^{+} e^{-}\right) / \Gamma_{\text {total }} \\
& \Gamma\left(K_{S}^{0} \rightarrow \pi^{0} e^{+} e^{-}\right) / \Gamma_{\text {total }} \\
& \Gamma\left(K_{S}^{0} \rightarrow \pi^{0} \mu^{+} \mu^{-}\right) / \Gamma_{\text {total }} \\
& \Gamma\left(K_{L}^{0} \rightarrow \mu^{+} \mu^{-}\right) / \Gamma_{\text {total }} \\
& \Gamma\left(K_{L}^{0} \rightarrow e^{+} e^{-}\right) / \Gamma_{\text {total }}
\end{aligned}
$$

$$
\begin{array}{ll} 
& (2.88 \pm 0.13) \times 10^{-7} \\
& (8.1 \pm 1.4) \times 10^{-8}(\mathrm{~S}=2.7) \\
& \left(1.5_{-0.9}^{+1.3}\right) \times 10^{-10} \\
& <4.3 \times 10^{-5}, \mathrm{CL}=90 \% \\
& <3.2 \times 10^{-7}, \mathrm{CL}=90 \% \\
& <1.4 \times 10^{-7}, \mathrm{CL}=90 \% \\
{[p] \quad} & \left(3.0_{-1.2}^{+1.5}\right) \times 10^{-9} \\
& \left(2.9_{-1.2}^{+1.5}\right) \times 10^{-9} \\
& (6.87 \pm 0.11) \times 10^{-9} \\
& \left(9_{-4}^{+6}\right) \times 10^{-12}
\end{array}
$$

HTTP://PDG.LBL.GOV 


$$
\begin{array}{lll}
\Gamma\left(K_{L}^{0} \rightarrow \pi^{+} \pi^{-} e^{+} e^{-}\right) / \Gamma_{\text {total }} & {[q]} & (3.11 \pm 0.19) \times 10^{-7} \\
\Gamma\left(K_{L}^{0} \rightarrow \pi^{0} \pi^{0} e^{+} e^{-}\right) / \Gamma_{\text {total }} & <6.6 \times 10^{-9}, \mathrm{CL}=90 \% \\
\Gamma\left(K_{L}^{0} \rightarrow \mu^{+} \mu^{-} e^{+} e^{-}\right) / \Gamma_{\text {total }} & (2.69 \pm 0.27) \times 10^{-9} \\
\Gamma\left(K_{L}^{0} \rightarrow e^{+} e^{-} e^{+} e^{-}\right) / \Gamma_{\text {total }} & (3.56 \pm 0.21) \times 10^{-8} \\
\Gamma\left(K_{L}^{0} \rightarrow \pi^{0} \mu^{+} \mu^{-}\right) / \Gamma_{\text {total }} & <3.8 \times 10^{-10}, \mathrm{CL}=90 \% \\
\Gamma\left(K_{L}^{0} \rightarrow \pi^{0} e^{+} e^{-}\right) / \Gamma_{\text {total }} & <2.8 \times 10^{-10}, \mathrm{CL}=90 \% \\
\Gamma\left(K_{L}^{0} \rightarrow \pi^{0} \nu \bar{\nu}\right) / \Gamma_{\text {total }} & <5.9 \times 10^{-7}, \mathrm{CL}=90 \% \\
\Gamma\left(\Sigma^{+} \rightarrow p e^{+} e^{-}\right) / \Gamma_{\text {total }} & <7 \times 10^{-6} \\
\Gamma\left(\Sigma^{+} \rightarrow p \mu^{+} \mu^{-}\right) / \Gamma_{\text {total }} & \left(9_{-8}^{+9}\right) \times 10^{-8}
\end{array}
$$

\section{$\Delta C=1$ WEAK NEUTRAL CURRENT FORBIDDEN}

Allowed by higher-order electroweak interactions.

$$
\begin{aligned}
& \Gamma\left(D^{+} \rightarrow \pi^{+} e^{+} e^{-}\right) / \Gamma_{\text {total }} \\
& \Gamma\left(D^{+} \rightarrow \pi^{+} \mu^{+} \mu^{-}\right) / \Gamma_{\text {total }} \\
& \Gamma\left(D^{+} \rightarrow \rho^{+} \mu^{+} \mu^{-}\right) / \Gamma_{\text {total }} \\
& \Gamma\left(D^{0} \rightarrow \gamma \gamma\right) / \Gamma_{\text {total }} \\
& \Gamma\left(D^{0} \rightarrow e^{+} e^{-}\right) / \Gamma_{\text {total }} \\
& \Gamma\left(D^{0} \rightarrow \mu^{+} \mu^{-}\right) / \Gamma_{\text {total }} \\
& \Gamma\left(D^{0} \rightarrow \pi^{0} e^{+} e^{-}\right) / \Gamma_{\text {total }} \\
& \Gamma\left(D^{0} \rightarrow \pi^{0} \mu^{+} \mu^{-}\right) / \Gamma_{\text {total }} \\
& \Gamma\left(D^{0} \rightarrow \eta e^{+} e^{-}\right) / \Gamma_{\text {total }} \\
& \Gamma\left(D^{0} \rightarrow \eta \mu^{+} \mu^{-}\right) / \Gamma_{\text {total }} \\
& \Gamma\left(D^{0} \rightarrow \pi^{+} \pi^{-} e^{+} e^{-}\right) / \Gamma_{\text {total }} \\
& \Gamma\left(D^{0} \rightarrow \rho^{0} e^{+} e^{-}\right) / \Gamma_{\text {total }} \\
& \Gamma\left(D^{0} \rightarrow \pi^{+} \pi^{-} \mu^{+} \mu^{-}\right) / \Gamma_{\text {total }} \\
& \Gamma\left(D^{0} \rightarrow \rho^{0} \mu^{+} \mu^{-}\right) / \Gamma_{\text {total }} \\
& \Gamma\left(D^{0} \rightarrow \omega e^{+} e^{-}\right) / \Gamma_{\text {total }} \\
& \Gamma\left(D^{0} \rightarrow \omega \mu^{+} \mu^{-}\right) / \Gamma_{\text {total }} \\
& \Gamma\left(D^{0} \rightarrow K^{-} K^{+} e^{+} e^{-}\right) / \Gamma_{\text {total }} \\
& \Gamma\left(D^{0} \rightarrow \phi e^{+} e^{-}\right) / \Gamma_{\text {total }} \\
& \Gamma\left(D^{0} \rightarrow K^{-} K^{+} \mu^{+} \mu^{-}\right) / \Gamma_{\text {total }} \\
& \Gamma\left(D^{0} \rightarrow \phi \mu^{+} \mu^{-}\right) / \Gamma_{\text {total }} \\
& \Gamma\left(D^{0} \rightarrow K^{-} \pi^{+} e^{+} e^{-}\right) / \Gamma_{\text {total }} \\
& \Gamma\left(D^{0} \rightarrow K^{-} \pi^{+} \mu^{+} \mu^{-}\right) / \Gamma_{\text {total }} \\
& \Gamma\left(D^{0} \rightarrow \pi^{+} \pi^{-} \pi^{0} \mu^{+} \mu^{-}\right) / \Gamma_{\text {total }} \\
& \Gamma\left(D_{s}^{+} \rightarrow K^{+} e^{+} e^{-}\right) / \Gamma_{\text {total }} \\
& \Gamma\left(D_{s}^{+} \rightarrow K^{+} \mu^{+} \mu^{-}\right) / \Gamma_{\text {total }} \\
& \Gamma\left(D_{s}^{+} \rightarrow K^{*}(892)^{+} \mu^{+} \mu^{-}\right) / \Gamma_{\text {total }} \\
&
\end{aligned}
$$

HTTP://PDG.LBL.GOV

$$
\begin{aligned}
& <7.4 \times 10^{-6}, \mathrm{CL}=90 \% \\
& <8.8 \times 10^{-6}, \mathrm{CL}=90 \% \\
& <5.6 \times 10^{-4}, \mathrm{CL}=90 \% \\
& <2.6 \times 10^{-5}, \mathrm{CL}=90 \% \\
& <1.2 \times 10^{-6}, \mathrm{CL}=90 \% \\
& <1.3 \times 10^{-6}, \mathrm{CL}=90 \% \\
& <4.5 \times 10^{-5}, \mathrm{CL}=90 \% \\
& <1.8 \times 10^{-4}, \mathrm{CL}=90 \% \\
& <1.1 \times 10^{-4}, \mathrm{CL}=90 \% \\
& <5.3 \times 10^{-4}, \mathrm{CL}=90 \% \\
& <3.73 \times 10^{-4}, \mathrm{CL}=90 \% \\
& <1.0 \times 10^{-4}, \mathrm{CL}=90 \% \\
& <3.0 \times 10^{-5}, \mathrm{CL}=90 \% \\
& <2.2 \times 10^{-5}, \mathrm{CL}=90 \% \\
& <1.8 \times 10^{-4}, \mathrm{CL}=90 \% \\
& <8.3 \times 10^{-4}, \mathrm{CL}=90 \% \\
& <3.15 \times 10^{-4}, \mathrm{CL}=90 \% \\
& <5.2 \times 10^{-5}, \mathrm{CL}=90 \% \\
& <3.3 \times 10^{-5}, \mathrm{CL}=90 \% \\
& <3.1 \times 10^{-5}, \mathrm{CL}=90 \% \\
& <3.85 \times 10^{-4}, \mathrm{CL}=90 \% \\
& <3.59 \times 10^{-4}, \mathrm{CL}=90 \% \\
& <8.1 \times 10^{-4}, \mathrm{CL}=90 \% \\
& <1.6 \times 10^{-3}, \mathrm{CL}=90 \% \\
& <3.6 \times 10^{-5}, \mathrm{CL}=90 \% \\
& <1.4 \times 10^{-3}, \mathrm{CL}=90 \% \\
& \\
& <\mathrm{cL}
\end{aligned}
$$

Page 23

Created: 5/1/2007 17:24 


$$
\Gamma\left(\Lambda_{c}^{+} \rightarrow p \mu^{+} \mu^{-}\right) / \Gamma_{\text {total }} \quad<3.4 \times 10^{-4}, \mathrm{CL}=90 \%
$$

\section{$\Delta B=1$ WEAK NEUTRAL CURRENT FORBIDDEN}

Allowed by higher-order electroweak interactions.

$$
\begin{aligned}
& \Gamma\left(B^{+} \rightarrow \pi^{+} e^{+} e^{-}\right) / \Gamma_{\text {total }} \\
& \Gamma\left(B^{+} \rightarrow \pi^{+} \mu^{+} \mu^{-}\right) / \Gamma_{\text {total }} \\
& \Gamma\left(B^{+} \rightarrow \pi^{+} \nu \bar{\nu}\right) / \Gamma_{\text {total }} \\
& \Gamma\left(B^{+} \rightarrow K^{+} e^{+} e^{-}\right) / \Gamma_{\text {total }} \\
& \Gamma\left(B^{+} \rightarrow K^{+} \mu^{+} \mu^{-}\right) / \Gamma_{\text {total }} \\
& \Gamma\left(B^{+} \rightarrow K^{+} \ell^{+} \ell^{-}\right) / \Gamma_{\text {total }} \\
& \Gamma\left(B^{+} \rightarrow K^{+} \bar{\nu} \nu\right) / \Gamma_{\text {total }} \\
& \Gamma\left(B^{+} \rightarrow K^{*}(892)^{+} e^{+} e^{-}\right) / \Gamma_{\text {total }} \\
& \Gamma\left(B^{+} \rightarrow K^{*}(892)^{+} \mu^{+} \mu^{-}\right) / \Gamma_{\text {total }} \\
& \Gamma\left(B^{+} \rightarrow K^{*}(892)^{+} \ell^{+} \ell^{-}\right) / \Gamma_{\text {total }} \\
& \Gamma\left(B^{0} \rightarrow \gamma \gamma\right) / \Gamma_{\text {total }} \\
& \Gamma\left(B^{0} \rightarrow e^{+} e^{-}\right) / \Gamma_{\text {total }} \\
& \Gamma\left(B^{0} \rightarrow \mu^{+} \mu^{-}\right) / \Gamma_{\text {total }} \\
& \Gamma\left(B^{0} \rightarrow K^{0} e^{+} e^{-}\right) / \Gamma_{\text {total }} \\
& \Gamma\left(B^{0} \rightarrow K^{0} \mu^{+} \mu^{-}\right) / \Gamma_{\text {total }} \\
& \Gamma\left(B^{0} \rightarrow K^{0} \ell^{+} \ell^{-}\right) / \Gamma_{\text {total }} \\
& \Gamma\left(B^{0} \rightarrow K^{*}(892)^{0} e^{+} e^{-}\right) / \Gamma_{\text {total }} \\
& \Gamma\left(B^{0} \rightarrow K^{*}(892)^{0} \mu^{+} \mu^{-}\right) / \Gamma_{\text {total }} \\
& \Gamma\left(B^{0} \rightarrow K^{*}(892)^{0} \nu \bar{\nu}\right) / \Gamma_{\text {total }} \\
& \Gamma\left(B^{0} \rightarrow K^{*}(892)^{0} \ell^{+} \ell^{-}\right) / \Gamma_{\text {total }} \\
& \Gamma\left(B^{0} \rightarrow \text { invisible }\right) / \Gamma_{\text {total }} \\
& \Gamma\left(B^{0} \rightarrow \nu \bar{\nu} \gamma\right) / \Gamma_{\text {total }} \\
& \Gamma\left(B \rightarrow s e^{+} e^{-}\right) / \Gamma_{\text {total }} \\
& \Gamma\left(B \rightarrow s \mu^{+} \mu^{-}\right) / \Gamma_{\text {total }} \\
& \Gamma\left(B \rightarrow s \ell^{+} \ell^{-}\right) / \Gamma_{\text {total }} \\
& \Gamma\left(B \rightarrow K e^{+} e^{-}\right) / \Gamma_{\text {total }} \\
& \Gamma\left(B \rightarrow K^{*}(892) e^{+} e^{-}\right) / \Gamma_{\text {total }} \\
& \Gamma\left(B \rightarrow K \mu^{+} \mu^{-}\right) / \Gamma_{\text {total }} \\
& \Gamma\left(B \rightarrow K^{*}(892) \mu^{+} \mu^{-}\right) / \Gamma_{\text {total }} \\
& \Gamma\left(B \rightarrow K \ell^{+} \ell^{-}\right) / \Gamma_{\text {total }} \\
& \Gamma\left(B \rightarrow K^{*}(892) \ell^{+} \ell^{-}\right) / \Gamma_{\text {total }} \\
& \Gamma\left(\bar{b} \rightarrow \mu^{+} \mu^{-} \text {anything }\right) / \Gamma_{\text {total }} \\
& \Gamma\left(B_{s}^{0} \rightarrow \gamma \gamma\right) / \Gamma_{\text {total }} \\
& \Gamma\left(B_{s}^{0} \rightarrow \mu^{+} \mu^{-}\right) / \Gamma_{\text {total }}
\end{aligned}
$$

HTTP://PDG.LBL.GOV

$$
\begin{aligned}
& <3.9 \times 10^{-3}, \mathrm{CL}=90 \% \\
& <9.1 \times 10^{-3}, \mathrm{CL}=90 \% \\
& <1.0 \times 10^{-4}, \mathrm{CL}=90 \% \\
& \left(8.0_{-1.9}^{+2.2}\right) \times 10^{-7}(\mathrm{~S}=1.4) \\
& \left(3.4_{-1.4}^{+1.9}\right) \times 10^{-7}(\mathrm{~S}=1.7) \\
& \text { [r] }(5.3 \pm 1.1) \times 10^{-7} \\
& <5.2 \times 10^{-5}, \mathrm{CL}=90 \% \\
& <4.6 \times 10^{-6}, \mathrm{CL}=90 \% \\
& <2.2 \times 10^{-6}, \mathrm{CL}=90 \% \\
& \text { [r] }<2.2 \times 10^{-6}, \mathrm{CL}=90 \% \\
& <6.2 \times 10^{-7}, \mathrm{CL}=90 \% \\
& <6.1 \times 10^{-8}, \mathrm{CL}=90 \% \\
& <3.9 \times 10^{-8}, \mathrm{CL}=90 \% \\
& <5.4 \times 10^{-7}, \mathrm{CL}=90 \% \\
& \left(2.0_{-1.0}^{+1.3}\right) \times 10^{-7}(\mathrm{~S}=1.6) \\
& \text { [r] }<6.8 \times 10^{-7}, \mathrm{CL}=90 \% \\
& <2.4 \times 10^{-6}, \mathrm{CL}=90 \% \\
& \left(1.22_{-0.32}^{+0.38}\right) \times 10^{-6} \\
& <1.0 \times 10^{-3}, \mathrm{CL}=90 \% \\
& \text { [r] }(1.17 \pm 0.30) \times 10^{-6} \\
& <2.2 \times 10^{-4}, \mathrm{CL}=90 \% \\
& <4.7 \times 10^{-5}, \mathrm{CL}=90 \% \\
& (4.7 \pm 1.3) \times 10^{-6} \\
& (4.3 \pm 1.2) \times 10^{-6} \\
& \text { [r] }(4.5 \pm 1.0) \times 10^{-6} \\
& \left(6.0_{-1.2}^{+1.4}\right) \times 10^{-7}(\mathrm{~S}=1.1) \\
& \left(1.24_{-0.32}^{+0.37}\right) \times 10^{-6} \\
& \left(4.7_{-1.0}^{+1.1}\right) \times 10^{-7} \\
& \left(1.19_{-0.29}^{+0.34}\right) \times 10^{-6} \\
& (5.4 \pm 0.8) \times 10^{-7} \\
& (1.05 \pm 0.20) \times 10^{-6} \\
& <3.2 \times 10^{-4}, \mathrm{CL}=90 \% \\
& <1.48 \times 10^{-4}, \mathrm{CL}=90 \% \\
& <1.5 \times 10^{-7}, \mathrm{CL}=90 \%
\end{aligned}
$$

Page 24 


$$
\begin{aligned}
& \Gamma\left(B_{s}^{0} \rightarrow e^{+} e^{-}\right) / \Gamma_{\text {total }} \\
& \Gamma\left(B_{s}^{0} \rightarrow \phi(1020) \mu^{+} \mu^{-}\right) / \Gamma_{\text {total }} \\
& \Gamma\left(B_{s}^{0} \rightarrow \phi \nu \bar{\nu}\right) / \Gamma_{\text {total }}
\end{aligned}
$$

$$
\begin{aligned}
& <5.4 \times 10^{-5}, \mathrm{CL}=90 \% \\
& <4.7 \times 10^{-5}, \mathrm{CL}=90 \% \\
& <5.4 \times 10^{-3}, \mathrm{CL}=90 \%
\end{aligned}
$$

\section{$\Delta T \equiv 1$ WEAK NEUTRAL CURRENT FORBIDDEN}

Allowed by higher-order electroweak interactions.
$\Gamma(t \rightarrow Z q(q=u, c)) / \Gamma_{\text {total }}$
[s] $\quad<13.7 \times 10^{-2}, \mathrm{CL}=95 \%$

\section{NOTES}

[a] $C$ parity forbids this to occur as a single-photon process.

[b] Time-reversal invariance requires this to be $0^{\circ}$ or $180^{\circ}$.

[c] Allowed by higher-order electroweak interactions.

[d] Violates $C P$ in leading order. Test of direct $C P$ violation since the indirect $C P$-violating and $C P$-conserving contributions are expected to be suppressed.

[e] $\operatorname{Re}\left(\epsilon^{\prime} / \epsilon\right)=\epsilon^{\prime} / \epsilon$ to a very good approximation provided the phases satisfy CPT invariance.

[f] Neglecting photon channels. See, e.g., A. Pais and S.B. Treiman, Phys. Rev. D12, 2744 (1975).

[g] Derived from measured values of $\phi_{+-}, \phi_{00},|\eta|,\left|m_{K_{L}^{0}}-m_{K_{S}^{0}}\right|$, and $\tau K_{S}^{0}$, as described in the introduction to "Tests of Conservation Laws."

[h] These two results are not independent, and both use the more precise measurement of $\left|q_{\bar{p}} / m_{\bar{p}}\right| /\left(q_{p} / m_{p}\right)$.

[i] The value is for the sum of the charge states or particle/antiparticle states indicated.

[j] A test of additive vs. multiplicative lepton family number conservation.

[k] The sign of $\Delta m_{32}^{2}$ is not known at this time. The range quoted is for the absolute value.

[/] Derived from an analysis of neutrino-oscillation experiments.

$[m]$ There is some controversy about whether nuclear physics and model dependence complicate the analysis for bound neutrons (from which the best limit comes). The first limit here is from reactor experiments with free neutrons.

$[n]$ This is the best limit for the mode $e^{-} \rightarrow \nu \gamma$. The best limit for "electron disappearance" is $6.4 \times 10^{24} \mathrm{yr}$. 
[o] This $D_{1}^{0}-D_{2}^{0}$ limit is inferred from the $D^{0}-\bar{D}^{0}$ mixing ratio $\Gamma\left(K^{+} \pi^{-}\right.$(via $\left.\left.\bar{D}^{0}\right)\right) / \Gamma\left(K^{-} \pi^{+}\right)$near the end of the $D^{0}$ Listings.

$[p]$ See the $K_{S}^{0}$ Particle Listings for the energy limits used in this measurement.

[q] See the $K_{L}^{0}$ Particle Listings for the energy limits used in this measurement.

$[r]$ An $\ell$ indicates an $e$ or a $\mu$ mode, not a sum over these modes.

$[s]$ This limit is for $\Gamma(t \rightarrow Z q) / \Gamma(t \rightarrow W b)$. 